c. 2

US Army Corps of Engineers Waterways Experiment Station
US-CE-C Property of the

United States Government

\title{
Leaky Coaxial Cable Sensor Slot/Sealant Configuration Performance Monitoring
}

by Larry N. Lynch

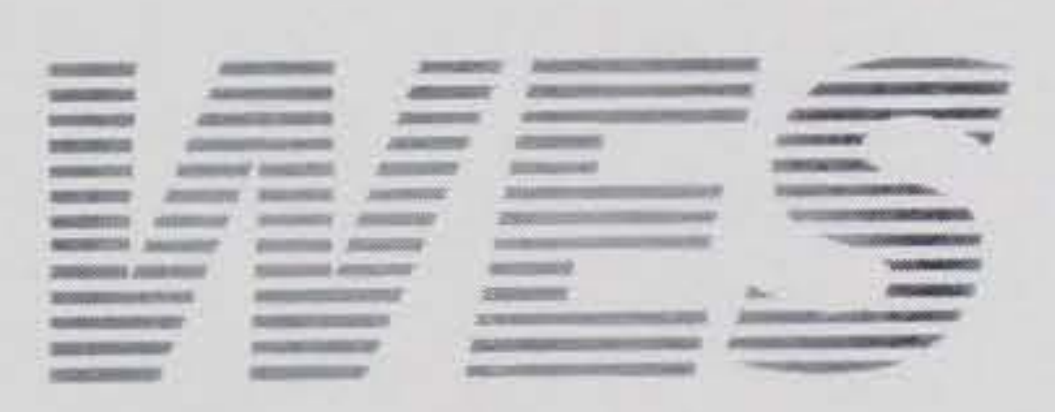

Approved For Public Release; Distribution Is Unlimited

Research Library

US Army Engineer Waterways

Experiment Station

Vicksburg. Milssissippi 


\section{Leaky Coaxial Cable Sensor Slot/Sealant Configuration Performance Monitoring}

by Larry N. Lynch

U.S. Army Corps of Engineers

Waterways Experiment Station

3909 Halls Ferry Road

Vicksburg, MS 39180-6199

Final report

Approved for public release; distribution is unlimited 


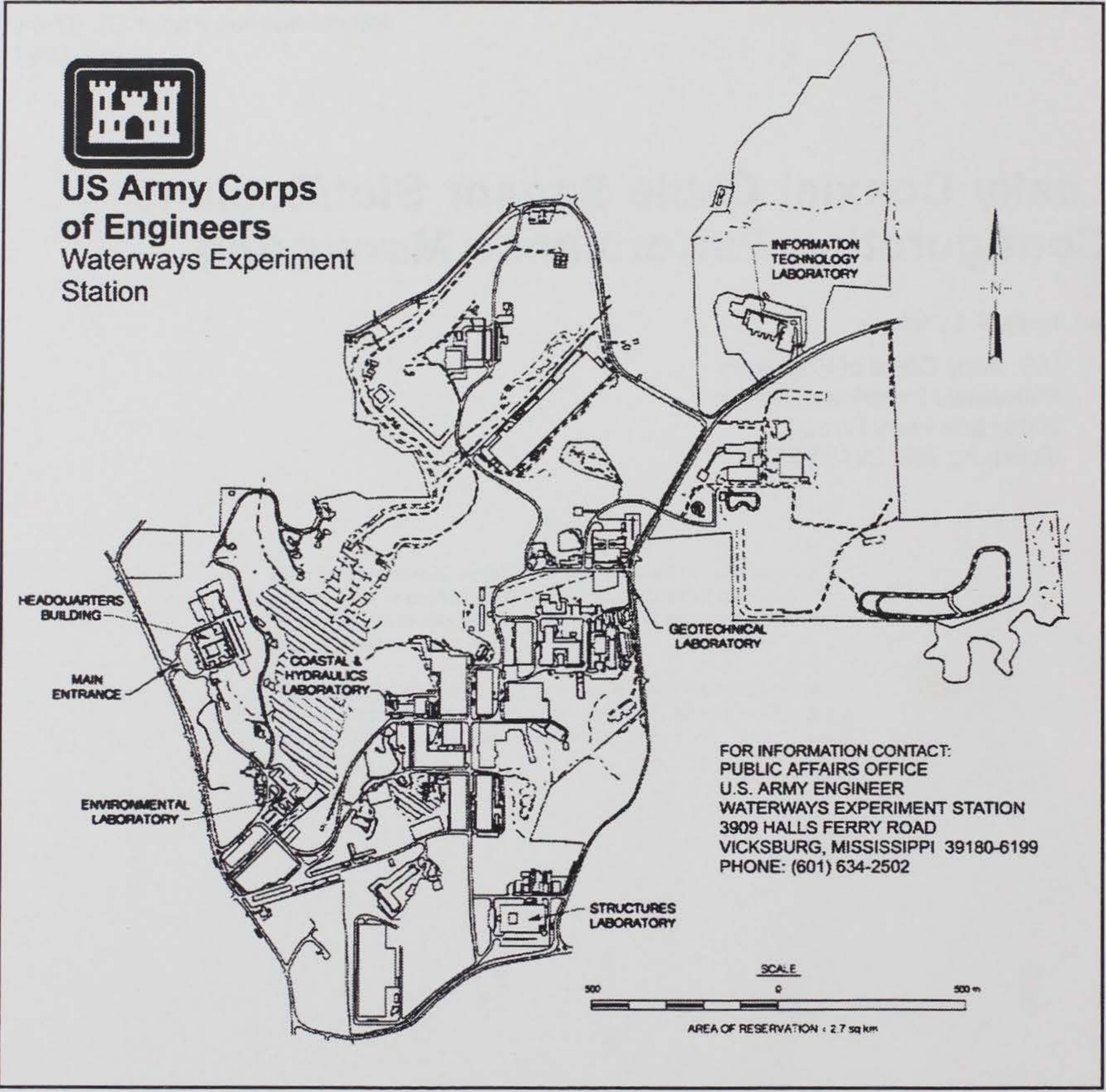

\section{Waterways Experiment Station Cataloging-in-Publication Data}

Lynch, Larry N.

Leaky coaxial cable sensor slot/sealant configuration performance monitoring / by Larry N. Lynch ; prepared for Department of the Air Force Electronic Security and Communications Center of Excellence.

46 p. : ill. ; $28 \mathrm{~cm}$. - (Miscellaneous paper ; GL-97-11)

Includes bibliographic references.

1. Coaxial cables - Testing. 2. Sealing compounds - Testing. I. United States. Army. Corps of Engineers. II. U.S. Army Engineer Waterways Experiment Station. III. Geotechnical Laboratory (U.S. Army Engineer Waterways Experiment Station) IV. United States. Air Force. V. Electronic Security and Communications Center of Excellence (U.S.) VI. Title. VII. Series: Miscellaneous paper (U.S. Army Engineer Waterways Experiment Station) ; GL-97-11.

TA7 W34m no.GL-97-11 


\section{Contents}

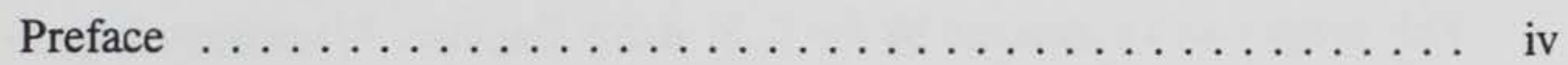

Conversion Factors, Non-SI to SI Units of Measurement $\ldots \ldots \ldots \ldots$ v

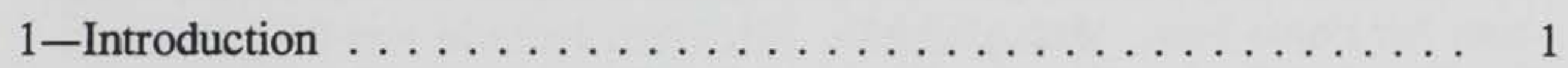

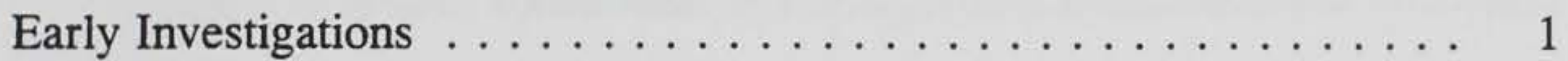

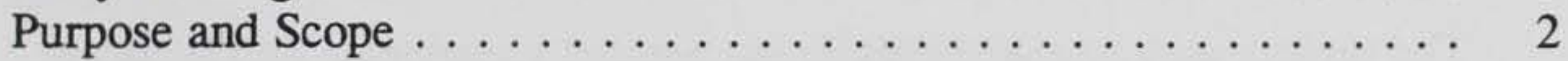

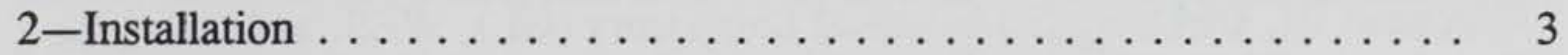

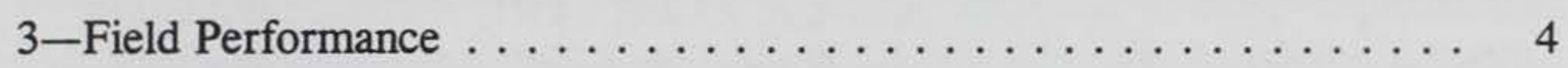

4 -Conclusions and Recommendations $\ldots \ldots \ldots \ldots \ldots$

SF 298 


\section{Preface}

This project was conducted by the U.S. Army Engineer Waterways Experiment Station (WES), Vicksburg, MS, during October 1996 and was sponsored by the Electronic Security and Communications Center of Excellence, Hanscom Air Force Base, Massachusetts. Mr. Ozell Johnson was the Electronic Security and Communications Center of Excellence technical monitor.

The project was conducted under the general supervision of Dr. W. F. Marcuson III, Director, Geotechnical Laboratory (GL), WES, and under the direct supervision of Dr. R. S. Rollings, Acting Chief, Airfields and Pavements Division (APD), and Mr. T. W. Vollor, Chief, Materials Analysis Branch (MAB), APD. The WES principal investigator and author of this report was Dr. Larry N. Lynch. The assistance of SMSG Mike Olenick, Westover Air Force Base, Massachusetts, during the field evaluation is gratefully acknowledged.

Dr. Robert W. Whalin was Director of WES and COL Bruce K. Howard, EN, was Commander during the preparation of this report. 


\section{Conversion Factors, Non-SI to SI Units of Measurement}

Non-SI units of measurement used in this report can be converted to SI units as follows:

\begin{tabular}{||l|l|l||}
\hline Multiply & By & To Obtain \\
\hline \hline Fahrenheit degrees & $5 / 9$ & Celsius degrees or kelvins \\
\hline inches & 25.4 & millimeters \\
\hline inches per inch per Fahrenheit degree & $5 / 9$ & $\begin{array}{l}\text { centimeters per centimeter per } \\
\text { Celsius degree }\end{array}$ \\
\hline pounds (force) per square inch & 6.894757 & kilopascals \\
\hline square inches & 6.4516 & square centimeters \\
\hline \hline $\begin{array}{l}\text { To obtain Celsius (C) temperature readings from Fahrenheit (F) readings, use the following } \\
\text { formula: } C=(5 / 9)(F-32) \text {. To obtain kelvin readings, use } K=(5 / 9)(F-32)+273.15 .\end{array}$ \\
\hline
\end{tabular}




\section{Introduction}

\section{Early Investigations}

In the early 1980 s evaluations were conducted on various joint sealant materials and potential slot configurations were considered for use with leaky coaxial cable sensor (LCCS) systems. During the early investigations, two of the main potential questions or concerns for sealant material selection and slot configuration included residual water accumulation in the slot during the performance life of the LCCS system, sealant material reactivity with the LCCS (i.e., would the system performance or sensitivity decrease due to reactions between various sealants and the cable) and the system being forced out of the slot resulting from thermal expansion of the cable. As a result of these concerns and the laboratory evaluations, a slot configuration and sealant application that consisted of complete encapsulation of the cable in the slot with Dow Corning ${ }^{\circledR} 888$ silicone sealant was selected. Figure 1 illustrates the slot/sealant configuration that was included in the guidelines for installing LCCS systems.

The slot/sealant configuration identified and incorporated into the installation guidelines has proven effective. However, new sealant materials are commercially available which were not originally included in the material evaluations and there is a tremendous interest in minimizing the cost of sensor cable installation. There are three potential methods of reducing the costs associated with sensor cable installation. The potential methods are:

a. Approve more than one sealant material for use in sensor cable installation thus allowing a contractor or installer to select the most economical material.

$b$. Reduce the labor requirements associated with the installation of the sealant material, i.e., allow the use of self-leveling sealants instead of nonsag sealants that require tooling to smooth the surface and to assist in adhering to the concrete.

c. Modify the slot/sealant configuration to reduce the amount of sealant required to seal the slot.

A research investigation was conducted to evaluate the three potential methods. The investigation is described by Malone, Lynch, and Godwin 
(1995). ${ }^{1}$ The main conclusions of the Malone, Lynch, and Godwin (1995) ${ }^{1}$ study that are relevant to this current program were:

a. Self-leveling silicone sealant materials could be used satisfactorily in the existing slot configuration.

$b$. If some moisture in the slot is not a problem, then a slot/sealant configuration similar to standard joint sealant procedures (see Figure 2) can be used, thus significantly reducing the amount of joint sealant material required to complete installation.

c. If a standard joint sealant configuration is used, then any joint sealant material can be used to seal the joint. Reactions between the cable and sealant would not be a concern because the sealant would not come in contact with the sealant.

$d$. The use of a standard joint sealant configuration would reduce the quantify of sealant required to seal the slot by approximately 60 to 75 percent. This would also reduce the material costs associated with sensor installation.

\section{Purpose and Scope}

The purpose of this report is to document the installation of a Ported Coaxial Cable Sensor (PCCS) system at Westover Air Force Base, Massachusetts, using the new slot/sealant configuration and to document the subsequent performance of the slot/sealant configuration approximately 2 years after installation.

1 Charles R. Malone, Larry N. Lynch, and Lenford N. Godwin. (1995). "Leaky coaxial cable sensor studies," Technical Report GL-95-17, U.S. Army Engineer Waterways Experiment Station, Vicksburg, MS. 


\section{Installation}

During June 1994, a new PCCS system was installed around the C-5 parking apron at Westover Air Force Base. The new installation was a replacement of an existing system that had been recently damaged by a contractor that was resealing the joints in the parking apron.

Since this was a reinstallation project, the slots were already cut into the pavement. The existing cable and Dow Corning ${ }^{\circledR} 888$ nonsag silicone joint sealant material were removed, the slots were cleaned, and the new cable, backer rod, and sealant were installed into the slot.

In standard joint resealing projects, the existing joint sealant material is typically removed by plowing or by sawing. The joint (or in this case the slot) would then be sandblasted to remove any remaining sealant material or residual concrete debris from the sawing operation. If sandblasting is not allowed in the area because of environmental concerns or the FOD potential for aircraft, wire brushing can be used to clean the slot. However, wire brushing will not effectively remove remaining joint sealant material, and the brushes must be inspected regularly for wear. When the wire brushes become worn, they will not remain in contact with the slot wall, and consequently the wall will not be properly cleaned. After sandblasting or wire brushing, the slots should be cleaned with compressed air to remove all loose debris remaining in the slot.

Once the slot has been cleaned with compressed air, the sensor cable should be inserted into the slot, backer rod should be inserted on top of the sensor cable, and then the sealant should be injected into the slot. The sealant material should be recessed below the pavement surface approximately $3 \mathrm{~mm}$ ( 0.125 in.). The small recess below the pavement surface will allow control point traffic (the point where authorized traffic enters the red line or secured area) to enter and exit without damaging the sealant material.

New slots (slots for a previously nonexistent sensor cable system) being cut into the concrete should be flushed or cleaned out with pressurized water to remove the residue from the sawing operation. Once the slot has become dry, it should be cleaned by sandblasting or wire brushing and the steps provided in the previous paragraph followed. 


\section{Field Performance}

During October 1996, a site visit was made to Westover Air Force Base to evaluate the field performance of the new slot/sealant configuration used on the C-5 parking apron. The PCCS system used in conjunction with the new slot/ sealant configuration was not operational. Initially, there was some concern that the nonoperational status of the PCCS may have been attributed to the new slot/sealant configuration. However, the Short Ported Coaxial Sensor (SPCS) system on the North apron was also nonoperational. This is significant from a slot/sealant configuration aspect because the North apron SPCS system was installed using the old slot/sealant configuration where the cable was completely encapsulated in Dow Corning ${ }^{\circledR} 888$. Communications with local security personnel indicated that the nonoperational status of both systems was due to a lack of routine maintenance of the system electronics. Additionally, since both systems were nonoperational and had been that way for a long period of time, the effect of the slot/sealant configuration on the operational status of sensor systems could not be determined.

The weather was cloudy and raining during the site visit. This made the evaluation of the joint sealant material easier. There was standing water on the C-5 parking apron. The condition in areas where the water was puddled over the new slot/sealant configuration were considered to be in good condition (i.e., the sealant was adhering to the concrete preventing the water from flowing from the surface into the joint). Some of the "puddled" areas were randomly evaluated to ensure that the sealant was adhering to the concrete instead of the slot being over full of water resulting in the puddling. All of the random evaluations indicated that the sealant was adhering to the concrete slot.

Areas of the sensor system that did not have standing water over them were inspected more closely. The dull appearance around the slot and the lack of standing water could potentially indicate that the sealant was not adhering to the concrete and water was infiltrating the slot. Approximately 50 percent of the interior slot (i.e., slot closest to the parked aircraft) next to the maintenance hangers exhibited the dull appearance. Inspection of these areas indicated that in most cases, the slot had been overfilled and therefore the sealant was extending above the standing water instead of the sealant not adhering to the concrete. A review of the installation inspection notes provides a potential cause of the overfilled slot. The interior slot was the first slot sealed using the Dow Corning ${ }^{\otimes} 890$ SL material. Therefore, this was the "learning" slot and some overfilling would be expected. The overall adhesion failure observed on the C-5 
parking apron was estimated to be less than 5 percent of the total linear footage of sealant. The small amount of adhesion failure was the only sealant deterioration noted during the field evaluation. The adhesion failures would allow some water to infiltrate the joint; however, the amount of water in the joint should not be significant and would not cause the joint to be flooded or completely filled with water.

The visual observations of the sealant performance in the new slot configuration indicate that the sealant had less than 5 percent adhesion failure, no cohesion failure, and no debris retention (i.e., rocks or debris embedded in the sealant). Therefore the sealant is performing satisfactorily. The exact cause of the adhesive failure could not be determined. Potential causes of joint sealant adhesive failure are improperly cleaned joints or insufficient sealant in the joint due to incorrect placement of the backer-rod material. It is expected that the small amount of adhesive failures at Westover Air Force Base can be attributed to joints that were not sufficiently cleaned. Most of the adhesive failures were noted at the beginning of the installation (i.e., where the installation crew began their cleaning process). The installation crew was very conscientious, but this project was the first time that they had used the new slot configuration and therefore they were learning the exact procedures required. As the installation crew became more familiar with the cleaning and installation techniques, the overall quality of the installation improved.

The overall condition of the sealant used in the new slot/sealant configuration was very good. However, to determine the applicability of the new slot/ sealant configuration, the climatic conditions to which the system was exposed must be defined. Weather data were obtained for time of sealant installation up to September 1996. Table 1 provides the monthly averages of maximum and minimum temperature, temperature extremes, total $24 \mathrm{hr}$ water equivalent (rainfall and snowfall), and total snowfall. The daily climatological data are provided in Appendix A.

Table 1 indicates that the sealant configuration was exposed to relatively severe winter conditions with respect to temperature and relatively high amounts of precipitation. Westover Air Force Base is located in an area bordering a wet-freeze and a wet-freeze-thaw climatic region (Peterson 1982). ${ }^{1}$ These two regions encompass approximately one-third of the United States. Silicone sealant materials exhibit a large linear viscoelastic region (Lynch 1996), ${ }^{2}$ which implies that the material properties of the sealant are not greatly affected by changes in temperature. In addition, the slots in which the cable systems are typically installed are not working joints. This means that there is no or only minimal movement at the slot due to environmental changes (expansion and contraction due to temperature or moisture variations). These two factors combined indicate that the performance of the sealant/slot configuration

1 Dale E. Petersen. (1982). "Resealing joints and cracks in rigid and flexible pavements," National Cooperative Highway Research Program Synthesis of Highway Practice 98, Transportation Research Board, National Research Council, Washington, DC.

2 Larry N. Lynch. (1996). "Rheological analysis of silicone pavement joint sealants," Technical Report GL-96-4, U.S. Army Engineer Waterways Experiment Station, Vicksburg, MS. 


\begin{tabular}{|c|c|c|c|c|c|c|}
\hline \multicolumn{7}{|c|}{$\begin{array}{l}\text { Table } 1 \\
\text { Average Monthly Climatological Data }\end{array}$} \\
\hline Data & $\begin{array}{l}\text { Average } \\
\text { Maximum } \\
{ }^{\circ} \mathrm{C}\left({ }^{\circ} \mathrm{F}\right) \\
\end{array}$ & $\begin{array}{l}\text { Average } \\
\text { Minimum } \\
{ }^{\circ} \mathrm{C}\left({ }^{\circ} \mathrm{F}\right) \\
\end{array}$ & $\begin{array}{l}\text { Extreme } \\
\text { Maximum } \\
{ }^{\circ} \mathrm{C}\left({ }^{\circ} \mathrm{F}\right) \\
\end{array}$ & $\begin{array}{l}\text { Extreme } \\
\text { Minimum } \\
{ }^{\circ} \mathrm{C}\left({ }^{\circ} \mathrm{F}\right) \\
\end{array}$ & $\begin{array}{l}\text { Total } \\
24 \text { Hour } \\
\text { Water Equivalent } \\
\mathrm{cm} \text { (in.) } \\
\end{array}$ & $\begin{array}{l}\text { Total } \\
24 \text { Hour } \\
\text { Snowfall } \\
\text { cm (in.) } \\
\end{array}$ \\
\hline August 1994 & $26.1(78.9)$ & $14.0(57.2)$ & $31(87)$ & $7(44)$ & $14.9(5.85)$ & $0.0(0.0)$ \\
\hline September 1994 & $22.3(72.2)$ & $9.9(49.8)$ & $29(85)$ & $3(38)$ & $11.6(4.57)$ & $0.0(0.0)$ \\
\hline October 1994 & $17.6(63.6)$ & $2.6(36.6)$ & $23(74)$ & $-4(25)$ & $3.8(1.49)$ & $0.0(0.0)$ \\
\hline November 1994 & $12.6(54.6)$ & $0.7(33.3)$ & $24(76)$ & $-9(15)$ & $10.9(4.30)$ & $8.4(3.3)$ \\
\hline December 1994 & $6.3(43.3)$ & $-4.6(23.8)$ & $17(63)$ & $-12(10)$ & $12.1(4.74)$ & $3.6(1.4)$ \\
\hline January 1995 & $3.3(37.9)$ & $-4.4(24.1)$ & $18(64)$ & $-14(07)$ & $8.6(3.39)$ & $10.9(4.3)$ \\
\hline February 1995 & $1.4(34.5)$ & $-9.2(15.5)$ & $12(53)$ & $-22(-08)$ & $7.2(2.84)$ & $23.6(9.3)$ \\
\hline March 1995 & $9.9(49.8)$ & $-0.9(30.4)$ & $21(69)$ & $-11(13)$ & $4.5(1.79)$ & $0.0(0.0)$ \\
\hline April 1995 & $13.6(56.4)$ & $0.9(33.6)$ & $23(74)$ & $-7(18)$ & $6.5(2.54)$ & $0.4(1.0)$ \\
\hline May 1995 & $20.4(68.7)$ & $6.5(43.7)$ & $31(87)$ & $-3(27)$ & $7.3(2.87)$ & $0.0(0.0)$ \\
\hline June 1995 & $27.3(81.2)$ & $12.0(53.6)$ & $36(97)$ & $4(39)$ & $3.5(1.36)$ & $0.0(0.0)$ \\
\hline July 1995 & $30.1(86.1)$ & $17.1(62.7)$ & $37(99)$ & $9(48)$ & $9.9(3.90)$ & $0.0(0.0)$ \\
\hline August 1995 & $28.8(83.8)$ & $13.1(55.6)$ & $21(96)$ & $4(40)$ & $9.0(3.55)$ & $0.0(0.0)$ \\
\hline September 1995 & $22.9(73.2)$ & $8.2(46.8)$ & $32(90)$ & $-1(30)$ & $9.7(3.83)$ & $0.0(0.0)$ \\
\hline October 1995 & $19.1(66.4)$ & $4.9(40.9)$ & $29(84)$ & $-1(30)$ & $25.9(10.2)$ & $0.0(0.0)$ \\
\hline November 1995 & $7.2(45.0)$ & $-2.0(28.4)$ & $18(64)$ & $-13(09)$ & $10.6(4.19)$ & $17.0(6.7)$ \\
\hline December 1995 & $0.5(33.0)$ & $-8.2(17.2)$ & $8(47)$ & $-19(-02)$ & $5.3(2.10)$ & $48.0(18.9)$ \\
\hline January 1996 & $0.3(32.5)$ & $-9.7(14.5)$ & $15(59)$ & $-23(-10)$ & $17.9(7.04)$ & $98.5(38.8)$ \\
\hline February 1996 & $1.6(34.9)$ & $-8.6(16.5)$ & $12(54)$ & $-28(-19)$ & $7.6(3.00)$ & $40.6(16.0)$ \\
\hline March 1996 & $6.5(43.7)$ & $-6.4(20.5)$ & $20(68)$ & $-22(-08)$ & $6.4(2.50)$ & $48.8(19.2)$ \\
\hline April 1996 & $14.3(57.8)$ & $2.3(36.2)$ & $28(83)$ & $-4(25)$ & $21.2(8.34)$ & $20.6(8.1)$ \\
\hline May 1996 & $19.6(67.2)$ & $5.9(42.6)$ & $33(92)$ & $-3(27)$ & $8.5(3.34)$ & $0.0(0.0)$ \\
\hline June 1996 & $25.1(77.1)$ & $13.1(55.6)$ & $30(86)$ & $4(39)$ & $6.5(2.56)$ & $0.0(0.0)$ \\
\hline July 1996 & $25.9(78.7)$ & $15.4(59.7)$ & $31(8)$ & $10(50)$ & $17.5(6.89)$ & $0.0(0.0)$ \\
\hline August 1996 & $27.5(81.5)$ & $15.3(59.6)$ & $32(90)$ & $8(46)$ & $3.8(1.48)$ & $0.0(0.0)$ \\
\hline September 1996 & $21.8(71.2)$ & $10.8(51.5)$ & $31(88)$ & $2(36)$ & $19.5(7.69)$ & $0.0(0.0)$ \\
\hline
\end{tabular}

should be similar when used in other locations throughout the United States. The performance prediction is based on the assumption that the slots are properly prepared and the sealant is properly installed. Additional evaluation sites could be selected in dry-freeze and dry-no freeze climatic regions to verify the sealant/slot configuration performance before policy changes are implemented if desired. 


\section{Conclusions and Recommendations}

The most recent sensor cable installation at Westover Air Force Base, Massachusetts, was installed using a modified sealant/slot configuration. The new sealant slot configuration reduced the required amount of joint sealant material by approximately 60 to 75 percent. The field evaluation of the new sealant/slot configuration after being in service for approximately 2 years indicates that the configuration performs satisfactorily. The main conclusions from this investigation are:

a. The new slot/sealant configuration is effective for the installation of sensor cable systems. This has been verified by the configuration surviving for 2 years in a harsh winter environment. The survivability of the this configuration in harsh winter climates should not be a major concern for future projects.

b. The Dow Corning ${ }^{\circledR} 890 \mathrm{SL}$ is effective in providing a seal for the cable slot.

The major recommendations from this investigation are:

a. The new slot/sealant configuration should be adopted for sensor cable installation in wet-freeze and wet-freeze thaw climatic regions.

b. Evaluation sites should be installed in other climatic regions to verify the new slot/sealant configuration for those regions.

c. Additional silicone sealant materials should be investigated for use for sensor cable installation.

d. Other types of joint sealant materials should be evaluated to allow alternatives to silicone materials if the user so desires. This could allow additional material cost savings to be realized. 


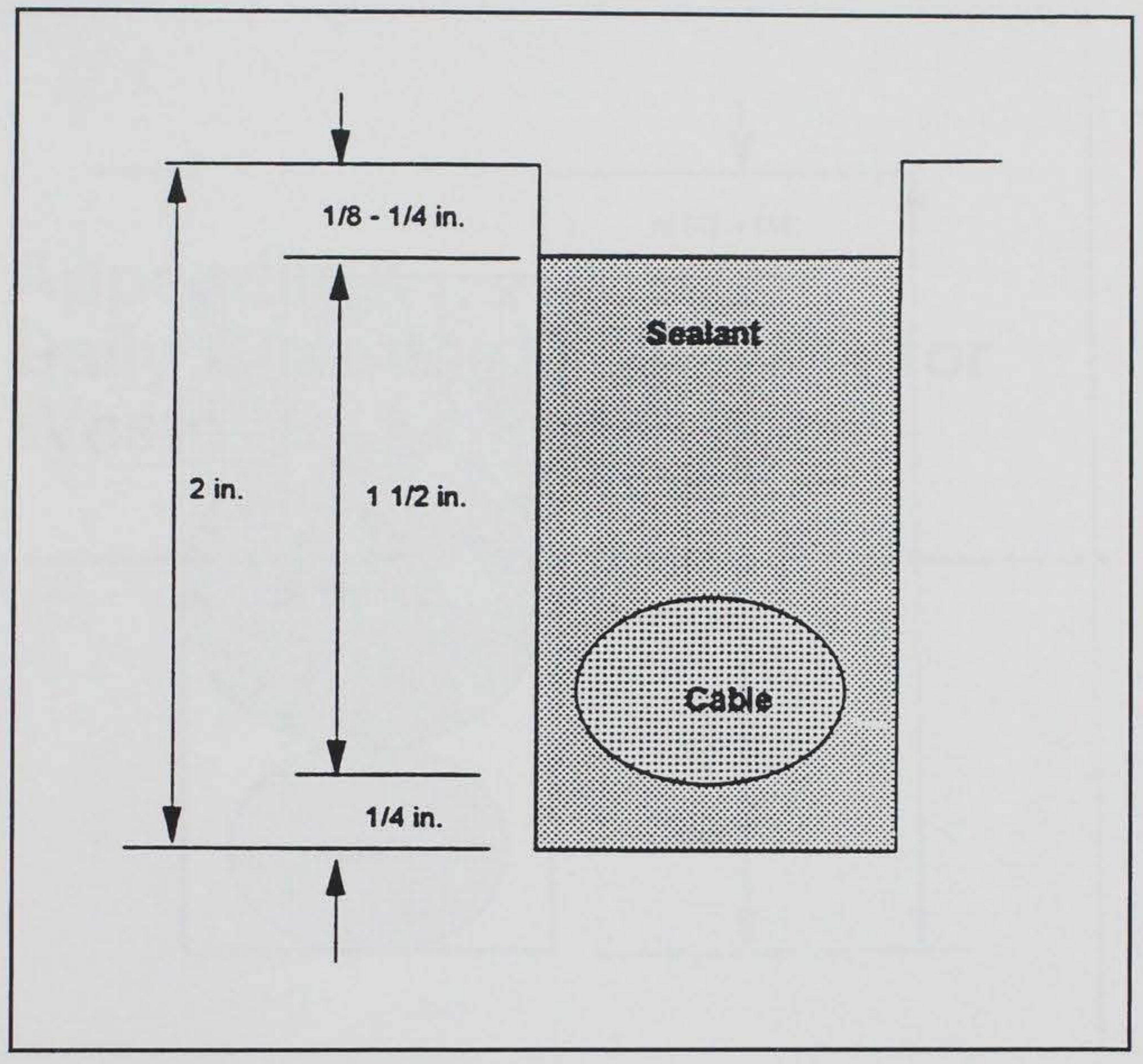

Figure 1. Standard cable-sealant (CS) configuration 


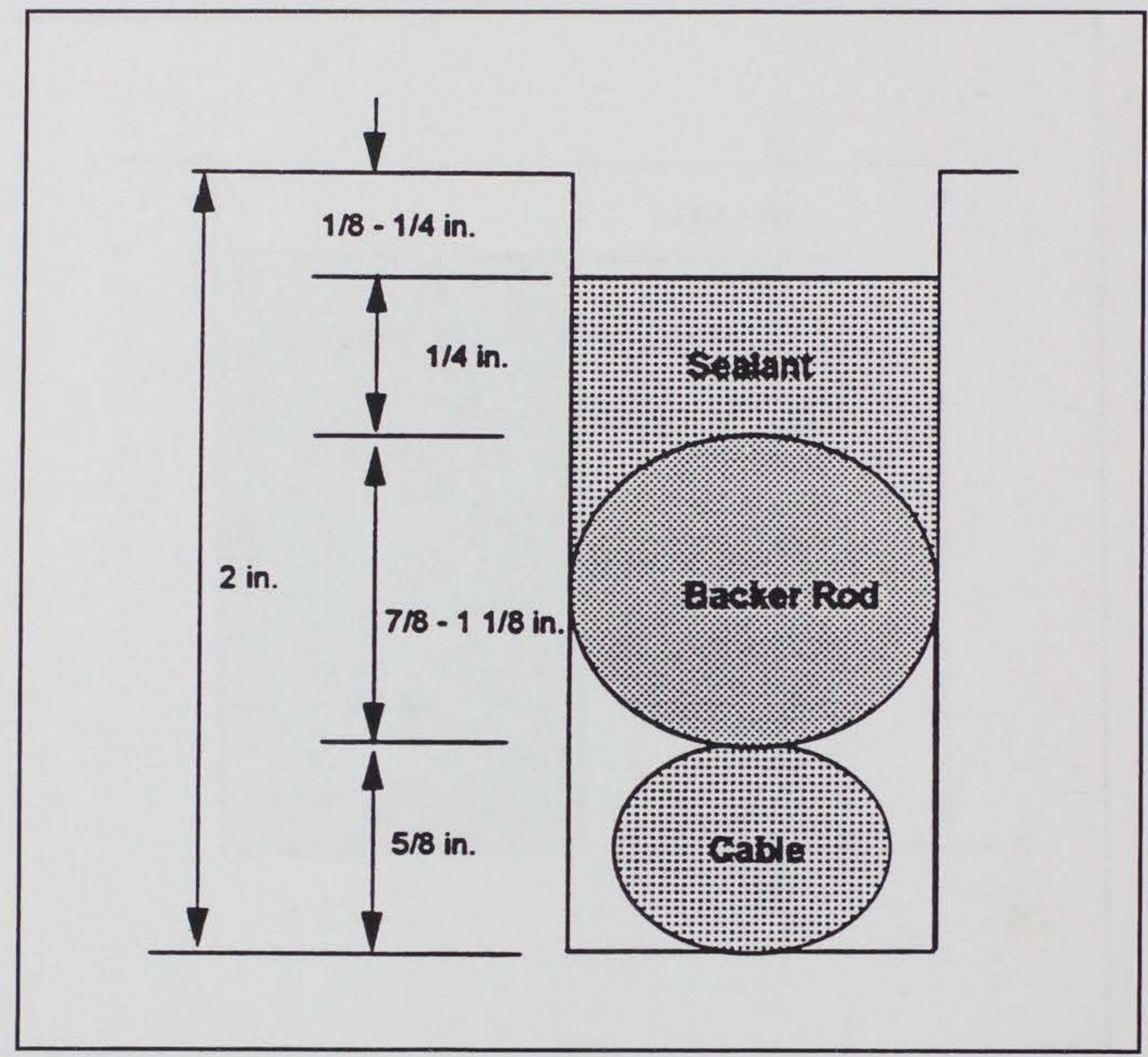

Figure 2. Proposed sealant-cable-backer rod configuration 


\section{Appendix A \\ Daily Climatological Data for Westover Air Force Base}




\begin{tabular}{|c|c|c|c|c|c|c|c|c|c|}
\hline \multicolumn{10}{|c|}{ Daily Climatological Data (Westover AFB, MA-August 1994) } \\
\hline \multirow[b]{3}{*}{ Day } & \multirow[b]{3}{*}{ Max } & \multirow[b]{3}{*}{ Min } & \multicolumn{3}{|c|}{ Temperature (F) } & \multicolumn{2}{|c|}{ Precipitation (in.) } & \multicolumn{2}{|c|}{ Max Wind } \\
\hline & & & \multirow{2}{*}{$\begin{array}{l}\text { Mean } \\
\text { (T) }\end{array}$} & \multicolumn{2}{|c|}{$\begin{array}{l}\text { Degree Days } \\
\left(\text { Base } 65^{\circ} \mathrm{F}\right) \\
\end{array}$} & \multirow{2}{*}{$\begin{array}{l}24 \mathrm{Hr} \text { Water } \\
\text { Equivalent }\end{array}$} & \multirow{2}{*}{$\begin{array}{l}24 \mathrm{Hr} \\
\text { Snowfall }\end{array}$} & \multirow{2}{*}{$\begin{array}{l}\text { Speed } \\
\text { (kts) }\end{array}$} & \multirow[b]{2}{*}{ Direction } \\
\hline & & & & Heating & Cooling & & & & \\
\hline 1 & 85 & 62 & 74 & & 9 & 0.00 & 0 & 12 & 17 \\
\hline 2 & 87 & 66 & 77 & & 12 & 0.00 & 0 & 11 & 23,21 \\
\hline 3 & 87 & 69 & 78 & & 13 & 0.02 & 0 & 11 & 22 \\
\hline 4 & 86 & 67 & 77 & & 12 & $T$ & 0 & 20 & 23 \\
\hline 5 & 77 & 54 & 66 & & 1 & 0.29 & 0 & 24 & 02 \\
\hline 6 & 73 & 49 & 61 & 4 & & 0.00 & 0 & 17 & 33 \\
\hline 7 & 78 & $45^{*}$ & 62 & 3 & & 0.00 & 0 & 8 & 22 \\
\hline 8 & 82 & 49 & 66 & & 1 & 0.00 & 0 & 9 & $21 / 29$ \\
\hline 9 & 85 & 53 & 69 & & 4 & 0.00 & 0 & 11 & 22 \\
\hline 10 & 80 & 61 & 71 & & 6 & 0.00 & 0 & 15 & 30 \\
\hline 11 & 72 & 55 & 64 & 1 & & 0.00 & 0 & 10 & 20 \\
\hline 12 & 79 & 63 & 71 & & 6 & 0.00 & 0 & 11 & 22 \\
\hline 13 & 85 & 70 & 78 & & 13 & 0.57 & 0 & 32 & 27 \\
\hline 14 & 81 & 62 & 72 & & 7 & 0.05 & 0 & 18 & $30 / 22$ \\
\hline 15 & 71 & 51 & 61 & 4 & & 0.00 & 0 & 18 & 27 \\
\hline 16 & 78 & 46 & 62 & 3 & & 0.00 & 0 & 14 & 24 \\
\hline 17 & 75 & 56 & 66 & & 1 & 0.13 & 0 & 11 & 21 \\
\hline 18 & 79 & 65 & 72 & & 7 & 2.28 & 0 & 13 & 35 \\
\hline 19 & 74 & 66 & 70 & & 5 & 0.14 & 0 & 7 & 02 \\
\hline 20 & 82 & 66 & 74 & & 9 & 0.00 & 0 & 13 & 20 \\
\hline 21 & 82 & 66 & 74 & & 9 & $1.04^{*}$ & 0 & 24 & 21 \\
\hline 22 & 67 & 58 & 63 & 2 & & $1.23^{*}$ & 0 & 17 & 03 \\
\hline 23 & 73 & 49 & 61 & 4 & & 0.00 & 0 & 14 & 01 \\
\hline 24 & 77 & $44^{* T}$ & 61 & 4 & & 0.00 & 0 & 7 & 21,24 \\
\hline 25 & 80 & 48 & 64 & 1 & & $T$ & 0 & 17 & 19,20 \\
\hline 26 & 84 & 60 & 72 & & 7 & 0.00 & 0 & 9 & 22,23 \\
\hline 27 & 85 & 61 & 73 & & 8 & 0.00 & 0 & 16 & 31 \\
\hline 28 & 86 & 58 & 72 & & 7 & 0.10 & 0 & 23 & 25 \\
\hline 29 & 75 & 52 & 64 & 1 & & I & 0 & 19 & 30 \\
\hline 30 & 74 & 49 & 62 & 3 & & 0.00 & 0 & 17 & 32 \\
\hline 31 & 67 & 53 & 60 & 5 & & $T$ & 0 & 7 & 20 \\
\hline Total & $\ldots$ & -- & $\ldots$ & 35 & 137 & 5.85 & 0 & $\ldots$ & $\ldots$ \\
\hline Mean & 78.9 & 57.2 & 68.3 & $\ldots$ & $\ldots$ & $\ldots$ & $\ldots$ & $\ldots$ & $\ldots$ \\
\hline Extreme & 87 & 44 & - & $\ldots$ & $\ldots$ & 2.28 & 0 & 32 & 27 \\
\hline
\end{tabular}




\begin{tabular}{|c|c|c|c|c|c|c|c|c|c|}
\hline \multicolumn{10}{|c|}{ Daily Climatological Data (Westover AFB, MA-September 1994) } \\
\hline \multirow[b]{3}{*}{ Day } & \multirow[b]{3}{*}{ Max } & \multirow[b]{3}{*}{ Min } & \multicolumn{3}{|c|}{ Temperature (F) } & \multicolumn{2}{|c|}{ Precipitation (in.) } & \multicolumn{2}{|c|}{ Max Wind } \\
\hline & & & \multirow[b]{2}{*}{ Mean $(T)$} & \multicolumn{2}{|c|}{$\begin{array}{l}\text { Degree Days } \\
\left(\text { Base } 65^{\circ} \mathrm{F}\right)\end{array}$} & \multirow{2}{*}{$\begin{array}{l}24 \mathrm{Hr} \text { Water } \\
\text { Equivalent }\end{array}$} & \multirow{2}{*}{$\begin{array}{l}24 \mathrm{Hr} \\
\text { Snowfall } \\
\end{array}$} & \multirow{2}{*}{$\begin{array}{l}\text { Speed } \\
\text { (kts) }\end{array}$} & \multirow[b]{2}{*}{ Direction } \\
\hline & & & & Heating & Cooling & & & & \\
\hline 1 & 77 & 48 & 63 & 2 & & 0.00 & 0 & 19 & 33 \\
\hline 2 & 70 & 42 & 56 & 9 & & 0.00 & 0 & 10 & 36 \\
\hline 3 & 71 & 38 & 55 & 10 & & 0.00 & 0 & 8 & $01,03,36$ \\
\hline 4 & 67 & 44 & 56 & 9 & & 0.00 & 0 & 17 & 01 \\
\hline 5 & 67 & 49 & 58 & 7 & & $T$ & 0 & 29 & 36 \\
\hline 6 & 74 & 48 & 61 & 4 & & 0.00 & 0 & 21 & 33 \\
\hline 7 & 78 & 43 & 61 & 4 & & 0.00 & 0 & 17 & 34 \\
\hline 8 & 78 & 48 & 64 & 1 & & 0.00 & 0 & $M$ & M \\
\hline 9 & 80 & 57 & 69 & & 4 & $T$ & 0 & $M$ & M \\
\hline 10 & 69 & 45 & 57 & 8 & & $T$ & 0 & $M$ & M \\
\hline 11 & 70 & 39 & 55 & 10 & & 0.00 & 0 & $M$ & M \\
\hline 12 & 76 & 43 & 60 & 5 & & 0.00 & 0 & $M$ & M \\
\hline 13 & 84 & 63 & 74 & 0 & 9 & $T$ & 0 & $M$ & M \\
\hline 14 & 78 & 58 & 68 & & 3 & $T$ & 0 & $M$ & M \\
\hline 15 & 76 & 48 & 62 & 3 & & 0.00 & 0 & $M$ & $\mathrm{M}$ \\
\hline 16 & 69 & 49 & 59 & 6 & & 0.02 & 0 & 17 & 16 \\
\hline 17 & 85 & 66 & 76 & & 11 & 0.61 & 0 & 24 & 28 \\
\hline 18 & 69 & 45 & 57 & 8 & & 0.26 & 0 & 23 & 34 \\
\hline 19 & 70 & 41 & 56 & 9 & & 0.00 & 0 & 28 & 27 \\
\hline 20 & 77 & 40 & 59 & 6 & & 0.00 & 0 & 17 & 28 \\
\hline 21 & 79 & 43 & 61 & 4 & & 0.00 & 0 & 10 & 18 \\
\hline 22 & 68 & 53 & 61 & 4 & & 0.20 & 0 & 18 & 05 \\
\hline 23 & 63 & 57 & 60 & 5 & & $2.14^{*}$ & 0 & 23 & $01 / 36$ \\
\hline 24 & 71 & 59 & 65 & $\ldots$ & $\ldots$ & $T$ & 0 & 13 & 34 \\
\hline 25 & 73 & 61 & 67 & $\ldots$ & 2 & 0.00 & 0 & 9 & 14.15 \\
\hline 26 & 65 & 62 & 64 & 1 & $\ldots$ & 0.22 & 0 & 16 & 08 \\
\hline 27 & 65 & 59 & 62 & 3 & - & 1.02 & 0 & 14 & 26 \\
\hline 28 & 72 & 53 & 63 & 2 & - & 0.10 & 0 & 16 & 22 \\
\hline 29 & 64 & 48 & 56 & 9 & $\ldots$ & $T$ & 0 & 25 & 27 \\
\hline 30 & 62 & 45 & 54 & 11 & $\ldots$ & 0.00 & 0 & 31 & 27 \\
\hline \multicolumn{10}{|l|}{31} \\
\hline Total & $\ldots$ & $\ldots$ & $\ldots$ & 140 & 29 & 4.57 & 0 & $\ldots$ & $\ldots$ \\
\hline Mean & 72.2 & 49.8 & 61.3 & -- & $\ldots$ & $\ldots$ & $\ldots$ & $\ldots$ & $\ldots$ \\
\hline Extreme & 85 & 38 & $\ldots$ & $\ldots$ & $\ldots$ & 2.14 & 0 & 31 & 27 \\
\hline
\end{tabular}




\begin{tabular}{|c|c|c|c|c|c|c|c|c|c|}
\hline \multicolumn{10}{|c|}{ Daily Climatological Data (Westover AFB, MA-October 1994) } \\
\hline \multirow[b]{3}{*}{ Day } & \multirow[b]{3}{*}{ Max } & \multirow[b]{3}{*}{ Min } & \multicolumn{3}{|c|}{ Temperature (F) } & \multicolumn{2}{|c|}{ Precipitation (in.) } & \multicolumn{2}{|c|}{ Max Wind } \\
\hline & & & \multirow[b]{2}{*}{ Mean $(T)$} & \multicolumn{2}{|c|}{$\begin{array}{l}\text { Degree Days } \\
\left.\text { (Base } 65^{\circ} \mathrm{F}\right)\end{array}$} & \multirow{2}{*}{$\begin{array}{l}24 \mathrm{Hr} \\
\text { Water } \\
\text { Equivalent } \\
\end{array}$} & \multirow{2}{*}{$\begin{array}{l}24 \mathrm{Hr} \\
\text { Snowfall } \\
\end{array}$} & \multirow{2}{*}{$\begin{array}{l}\text { Speed } \\
\text { (kts) }\end{array}$} & \multirow[b]{2}{*}{ Direction } \\
\hline & & & & Heating & Cooling & & & & \\
\hline 1 & 56 & 37 & 47 & 18 & & 0.10 & 0 & 14 & 01,23 \\
\hline 2 & 59 & 38 & 49 & 16 & & 0.00 & 0 & 27 & 29 \\
\hline 3 & 60 & 30 & 45 & 20 & & 0.00 & 0 & 22 & 34 \\
\hline 4 & 60 & 45 & 53 & 12 & & 0.00 & 0 & 21 & 32,35 \\
\hline 5 & 57 & 33 & 45 & 20 & & 0.00 & 0 & 23 & 30 \\
\hline 6 & 61 & 33 & 47 & 18 & & 0.00 & 0 & 20 & 31 \\
\hline 7 & 69 & 32 & 51 & 14 & & 0.00 & 0 & 14 & 19 \\
\hline 8 & 74 & 40 & 54 & 11 & & 0.00 & 0 & 20 & 13 \\
\hline 9 & 74 & 51 & 63 & 2 & & 0.31 & 0 & 28 & 31 \\
\hline 10 & 59 & 34 & 47 & 18 & & 0.00 & 0 & 32 & 30 \\
\hline 11 & 58 & 28 & 43 & 22 & & 0.00 & 0 & 28 & 35 \\
\hline 12 & 61 & 25 & 43 & 22 & & 0.00 & 0 & 11 & 27 \\
\hline 13 & 66 & 28 & 47 & 18 & & 0.00 & 0 & 13 & 19 \\
\hline 14 & 65 & 35 & 50 & 15 & & 0.00 & 0 & 16 & 34 \\
\hline 15 & 60 & 35 & 48 & 17 & & 0.00 & 0 & 21 & 35 \\
\hline 16 & 66 & 30 & 48 & 17 & & 0.00 & 0 & 24 & 01 \\
\hline 17 & 66 & 29 & 48 & 17 & & 0.00 & 0 & 13 & 34 \\
\hline 18 & 62 & 32 & 47 & 18 & & 0.03 & 0 & 10 & 20,19 \\
\hline 19 & 66 & 50 & 58 & 7 & & $T$ & 0 & 10 & 17,14 \\
\hline 20 & 64 & 55 & 60 & 5 & & 0.48 & 0 & 11 & 21 \\
\hline 21 & 69 & 51 & 60 & 5 & & 0.00 & 0 & 25 & 35 \\
\hline 22 & 72 & 45 & 59 & 6 & & 0.00 & 0 & 21 & 34 \\
\hline 23 & 59 & 39 & 49 & 16 & & 0.43 & 0 & 7 & 33,34 \\
\hline 24 & 66 & 40 & 53 & 12 & & 0.00 & 0 & 18 & 25 \\
\hline 25 & 65 & 37 & 51 & 14 & & 0.00 & 0 & 14 & 04 \\
\hline 26 & 57 & 32 & 45 & 20 & & 0.00 & 0 & 10 & 30 \\
\hline 27 & 55 & 30 & 43 & 22 & & 0.00 & 0 & 17 & 36 \\
\hline 28 & 62 & 27 & 45 & 20 & & 0.00 & 0 & 16 & 18 \\
\hline 29 & 65 & 34 & 50 & 15 & & 0.00 & 0 & 22 & $17,18,19,20$ \\
\hline 30 & 71 & 37 & 54 & 11 & & 0.00 & 0 & 13 & 20 \\
\hline 31 & 68 & 43 & 56 & 9 & & 0.14 & 0 & 17 & 18 \\
\hline Total & $\ldots$ & $\ldots$ & $\ldots$ & 457 & 0 & 1.49 & 0 & $\ldots$ & $\ldots$ \\
\hline Mean & 63.6 & 36.6 & 50.2 & $\ldots$ & $\ldots$ & $\ldots$ & $\ldots$ & $\ldots$ & $\ldots$ \\
\hline Extreme & 74 & 25 & - & $\ldots$ & $\ldots$ & 0.48 & 0 & 32 & 30 \\
\hline
\end{tabular}




\begin{tabular}{|c|c|c|c|c|c|c|c|c|c|}
\hline \multicolumn{10}{|c|}{ Daily Climatological Data (Westover AFB, MA-November 1994) } \\
\hline \multirow[b]{3}{*}{ Day } & \multirow[b]{3}{*}{ Max } & \multirow[b]{3}{*}{ Min } & \multicolumn{3}{|c|}{ Temperature (F) } & \multicolumn{2}{|c|}{ Precipitation (in.) } & \multicolumn{2}{|c|}{ Max Wind } \\
\hline & & & \multirow[b]{2}{*}{ Mean (T) } & \multicolumn{2}{|c|}{$\begin{array}{l}\text { Degree Days } \\
\left(\text { Base } 65^{\circ} \mathrm{F}\right) \\
\end{array}$} & \multirow{2}{*}{$\begin{array}{l}24 \mathrm{Hr} \\
\text { Water } \\
\text { Equivalent } \\
\end{array}$} & \multirow{2}{*}{$\begin{array}{l}24 \mathrm{Hr} \\
\text { Snowfall } \\
\end{array}$} & \multirow{2}{*}{$\begin{array}{l}\text { Speed } \\
\text { (kts) } \\
\end{array}$} & \multirow[b]{2}{*}{ Direction } \\
\hline & & & & Heating & Cooling & & & & \\
\hline 1 & 67 & 54 & 61 & 4 & & 0.33 & 0.0 & 21 & 18 \\
\hline 2 & 57 & 45 & 51 & 14 & & 0.03 & 0.0 & 40 & 28 \\
\hline 3 & 66 & 33 & 50 & 15 & & 0.00 & $\begin{array}{l}0.0 \\
\end{array}$ & 30 & 33 \\
\hline 4 & $76^{*}$ & 42 & 59 & 6 & & 0.00 & 0.0 & 19 & 16,19 \\
\hline 5 & $74^{*}$ & 49 & 62 & 3 & & 0.00 & 0.0 & 16 & 18,17 \\
\hline 6 & 64 & 44 & 54 & 11 & & 0.29 & 0.0 & 38 & 27 \\
\hline 7 & 55 & 40 & 48 & 17 & & $T$ & 0.0 & 40 & 29 \\
\hline 8 & 64 & 39 & 52 & 13 & & 0.00 & 0.0 & 20 & 21 \\
\hline 9 & 62 & 47 & 55 & 10 & & 0.03 & 0.0 & 18 & 01 \\
\hline 10 & 54 & 37 & 46 & 19 & & 0.22 & 0.0 & 29 & 33,30 \\
\hline 11 & 46 & 29 & 38 & 27 & & 0.00 & 0.0 & 25 & 34 \\
\hline 12 & 51 & 22 & 37 & 28 & & 0.00 & 0.0 & 16 & 17 \\
\hline 13 & 59 & 32 & 46 & 19 & & 0.00 & 0.0 & 18 & 02,03 \\
\hline 14 & 59 & 28 & 44 & 21 & & 0.00 & 0.0 & 20 & 19,20 \\
\hline 15 & 65 & 44 & 55 & 10 & & 0.00 & 0.0 & 24 & 32 \\
\hline 16 & 48 & 33 & 41 & 24 & & 0.00 & 0.0 & 17 & 03 \\
\hline 17 & 58 & 27 & 43 & 22 & & 0.00 & 0.0 & 17 & 06 \\
\hline 18 & 53 & 36 & 45 & 20 & & 0.07 & 0.0 & 15 & 34 \\
\hline 19 & 57 & 42 & 50 & 15 & & $T$ & 0.0 & 23 & 28 \\
\hline 20 & 49 & 28 & 39 & 26 & & 0.00 & 0.0 & 22 & 36 \\
\hline 21 & 53 & 25 & 39 & 26 & & $1.72^{*}$ & 0.0 & 20 & 30 \\
\hline 22 & 55 & 35 & 45 & 20 & & $T$ & 0.0 & 35 & 30 \\
\hline 23 & 41 & 23 & 32 & 33 & & $T$ & 0.1 & 37 & 30 \\
\hline 24 & 33 & 20 & 27 & 38 & & 0.00 & 0.0 & 27 & 29 \\
\hline 25 & 50 & 27 & 39 & 26 & & 0.00 & 0.0 & 24 & 26 \\
\hline 26 & 41 & 20 & 31 & 34 & & 0.00 & 0.0 & 31 & 28 \\
\hline 27 & 35 & 15 & 25 & 40 & & 0.20 & 2.2 & 19 & 08 \\
\hline 28 & 45 & 31 & 38 & 27 & & $1.41^{*}$ & 1.0 & 16 & 08,09 \\
\hline 29 & 52 & 28 & 40 & 25 & & 0.00 & 0.0 & 23 & 24 \\
\hline 30 & 49 & 24 & 37 & 28 & & $T$ & 0.0 & 26 & 28 \\
\hline 31 & $\ldots$ & $\ldots$ & $\ldots$ & $\ldots$ & & $\ldots$ & $\ldots$ & $\ldots$ & $\ldots$ \\
\hline Total & $\ldots$ & $\ldots$ & $\ldots$ & 621 & 0 & 4.30 & 3.3 & $\ldots$ & $\ldots$ \\
\hline Mean & 54.6 & 33.3 & 44.3 & $\ldots$ & $\ldots$ & $\ldots$ & $\ldots$ & $\ldots$ & $\ldots$ \\
\hline Extreme & 76 & 15 & $\ldots$ & $\ldots$ & $\ldots$ & 1.72 & 2.2 & 40 & 28,29 \\
\hline
\end{tabular}




\begin{tabular}{|c|c|c|c|c|c|c|c|c|c|}
\hline \multicolumn{10}{|c|}{ Daily Climatological Data (Westover AFB, MA-December 1994) } \\
\hline \multirow[b]{3}{*}{ Day } & \multirow[b]{3}{*}{ Max } & \multirow[b]{3}{*}{ Min } & \multicolumn{3}{|c|}{ Temperature (F) } & \multicolumn{2}{|c|}{ Precipitation (in.) } & \multicolumn{2}{|c|}{ Max Wind } \\
\hline & & & \multirow[b]{2}{*}{ Mean $(T)$} & \multicolumn{2}{|c|}{$\begin{array}{l}\text { Degree Days } \\
\text { (Base } 65^{\circ} \mathrm{F} \text { ) }\end{array}$} & \multirow{2}{*}{$\begin{array}{l}24 \mathrm{Hr} \\
\text { Water } \\
\text { Equivalent } \\
\end{array}$} & \multirow{2}{*}{$\begin{array}{l}24 \mathrm{Hr} \\
\text { Snowfall } \\
\end{array}$} & \multirow{2}{*}{$\begin{array}{l}\text { Speed } \\
\text { (kts) }\end{array}$} & \multirow[b]{2}{*}{ Direction } \\
\hline & & & & Heating & Cooling & & & & \\
\hline 1 & 43 & 21 & 32 & 33 & & 0.00 & 0.0 & 20 & 33 \\
\hline 2 & 55 & 25 & 40 & 25 & & 0.00 & 0.0 & 26 & 20,18 \\
\hline 3 & $58 * T$ & 26 & 42 & 33 & & 0.00 & 0.0 & 9 & 20 \\
\hline 4 & 63 & 28 & 46 & 19 & & 0.00 & 0.0 & 16 & 28 \\
\hline 5 & 48 & 38 & 43 & 22 & & 1.61 & 0.0 & 14 & 33 \\
\hline 6 & 56 & 45 & 51 & 14 & & 0.00 & 0.0 & 22 & 28 \\
\hline 7 & 52 & 30 & 41 & 24 & & 0.14 & 0.2 & 22 & 30 \\
\hline 8 & 33 & 20 & 27 & 38 & & 0.00 & 0.0 & 29 & 35,34 \\
\hline 9 & 35 & 15 & 25 & 40 & & 0.04 & 0.3 & 13 & 25.36 \\
\hline 10 & 39 & 33 & 36 & 39 & & 0.64 & 0.5 & 22 & 30 \\
\hline 11 & 41 & 25 & 33 & 32 & & 0.30 & 0.0 & 34 & 29 \\
\hline 12 & 25 & 14 & 20 & 45 & & 0.00 & 0.0 & 29. & 33 \\
\hline 13 & 25 & 10 & 18 & 47 & & 0.00 & 0.0 & 21 & 34 \\
\hline 14 & 33 & 26 & 30 & 35 & & $\mathrm{~T}$ & $T$ & 20 & 03 \\
\hline 15 & 38 & 19 & 29 & 36 & & 0.00 & 0.0 & 20 & 33 \\
\hline 16 & 31 & 16 & 24 & 41 & & $\mathrm{~T}$ & $\mathrm{~T}$ & 15 & 34,01 \\
\hline 17 & 35 & 30 & 33 & 32 & & 0.24 & 0.4 & 12 & 35,11 \\
\hline 18 & 43 & 27 & 35 & 40 & & 0.06 & 0.0 & 15 & 36 \\
\hline 19 & 38 & 22 & 30 & 35 & & 0.00 & 0.0 & 27 & 33 \\
\hline 20 & 40 & 15 & 28 & 37 & & 0.00 & 0.0 & 15 & 30 \\
\hline 21 & 53 & 21 & 37 & 28 & & 0.00 & 0.0 & 14 & 20 \\
\hline 22 & 55 & 23 & 39 & 26 & & 0.00 & 0.0 & 8 & 30,29 \\
\hline 23 & 50 & 25 & 38 & 27 & & 0.27 & 0.0 & 37 & 35 \\
\hline 24 & 48 & 42 & 45 & 20 & & $1.34^{*}$ & 0.0 & 45 & 04 \\
\hline 25 & 55 & 37 & 46 & 19 & & $T$ & 0.0 & 27 & 01 \\
\hline 26 & 48 & 23 & 36 & 29 & & 0.00 & 0.0 & 21 & 35 \\
\hline 27 & 44 & 18 & 31 & 34 & & 0.00 & 0.0 & 13 & 21 \\
\hline 28 & 50 & 23 & 37 & 28 & & 0.01 & 0.0 & 30 & 28 \\
\hline 29 & 43 & 14 & 29 & 36 & & $T$ & 0.0 & 43 & 34 \\
\hline 30 & 29 & 12 & 21 & 44 & & 0.00 & 0.0 & 27 & 34 \\
\hline 31 & 35 & 13 & 24 & 41 & & 0.09 & 0.0 & 19 & 17 \\
\hline Total & $\ldots$ & $\ldots$ & $\ldots$ & 999 & & 4.74 & 1.4 & $\ldots$ & $\ldots$ \\
\hline Mean & 43.3 & 23.8 & 33.8 & $\ldots$ & $\ldots$ & $\ldots$ & $\ldots$ & $\ldots$ & $\ldots$ \\
\hline Extreme & 63 & 10 & $\ldots$ & $\ldots$ & $\ldots$ & 1.34 & 0.5 & 45 & 04 \\
\hline
\end{tabular}




\begin{tabular}{|c|c|c|c|c|c|c|c|c|c|}
\hline \multicolumn{10}{|c|}{ Daily Climatological Data (Westover AFB, MA-January 1995) } \\
\hline \multirow[b]{3}{*}{ Day } & \multirow[b]{3}{*}{ Max } & \multirow[b]{3}{*}{ Min } & \multicolumn{3}{|c|}{ Temperature (F) } & \multicolumn{2}{|c|}{ Precipitation (in.) } & \multicolumn{2}{|c|}{ Max Wind } \\
\hline & & & \multirow[b]{2}{*}{ Mean (T) } & \multicolumn{2}{|c|}{$\begin{array}{l}\text { Degree Days } \\
\left.\text { (Base } 65^{\circ} \mathrm{F}\right)\end{array}$} & \multirow{2}{*}{$\begin{array}{l}24 \mathrm{Hr} \\
\text { Water } \\
\text { Equivalent }\end{array}$} & \multirow{2}{*}{$\begin{array}{l}24 \mathrm{Hr} \\
\text { Snowfall }\end{array}$} & \multirow{2}{*}{$\begin{array}{l}\text { Speed } \\
\text { (kts) } \\
\end{array}$} & \multirow[b]{2}{*}{ Direction } \\
\hline & & & & Heating & Cooling & & & & \\
\hline 1 & 41 & 32 & 37 & 28 & $\ldots$ & 0.56 & 0.0 & 10 & 16 \\
\hline 2 & 35 & 26 & 31 & 34 & $\ldots$ & 0.29 & 1.0 & 39 & 29 \\
\hline 3 & 31 & 21 & 26 & 39 & $\ldots$ & 0.00 & 0.0 & 23 & 27 \\
\hline 4 & 30 & 15 & 23 & 42 & $\ldots$ & $T$ & $T$ & 27 & 30 \\
\hline 5 & 25 & 11 & 18 & 47 & $\ldots$ & 0.00 & 0.0 & 24 & 27,28 \\
\hline 6 & 36 & 15 & 26 & 39 & -- & 0.09 & $T$ & 25 & 21,20 \\
\hline 7 & 44 & 31 & 38 & 27 & $\ldots$ & 0.64 & 0.0 & 26 & 30 \\
\hline 8 & 35 & 17 & 26 & 39 & $\ldots$ & $\mathrm{T}$ & $T$ & 20 & 36 \\
\hline 9 & 35 & 12 & 24 & 41 & $\ldots$ & 0.00 & 0.0 & 22 & 29 \\
\hline 10 & 29 & 9 & 19 & 46 & $\ldots$ & 0.00 & 0.0 & 18 & 33 \\
\hline 11 & 19 & 13 & 16 & 49 & $\ldots$ & 0.10 & 0.8 & 15 & 35 \\
\hline 12 & 31 & 19 & 25 & 40 & $\ldots$ & 0.28 & 1.2 & 13 & 36 \\
\hline 13 & 43 & 32 & 38 & 27 & -- & 0.02 & 0.0 & 7 & 34,33 \\
\hline 14 & 54 & 35 & 45 & 20 & $\ldots$ & 0.08 & 0.0 & 12 & 18 \\
\hline 15 & $64^{*}$ & 46 & 55 & 10 &.- & 0.19 & 0.0 & 24 & 17,19 \\
\hline 16 & $60^{*}$ & 52 & 56 & 9 & $\ldots$ & 0.21 & 0.0 & 21 & 19,18 \\
\hline 17 & 52 & 39 & 46 & 19 & -- & 0.03 & 0.0 & 26 & 35 \\
\hline 18 & 39 & 34 & 37 & 28 & $\ldots$ & 0.02 & 0.0 & 20 & 06 \\
\hline 19 & 38 & 33 & 36 & 29 & $\ldots$ & 0.02 & 0.0 & 13 & 02 \\
\hline 20 & 42 & 36 & 39 & 26 & $\ldots$ & 0.58 & 0.0 & 30 & 02 \\
\hline 21 & 42 & 37 & 40 & 25 & $\ldots$ & 0.06 & 0.0 & 23 & 16 \\
\hline 22 & 37 & 33 & 35 & 30 & -- & 0.14 & $\mathrm{~T}$ & 17 & 28 \\
\hline 23 & 35 & 28 & 32 & 33 & - & 0.06 & 1.0 & 18 & 30,27 \\
\hline 24 & 35 & 30 & 33 & 32 & $\ldots$ & $T$ & 0.1 & 25 & 36 \\
\hline 25 & 35 & 21 & 28 & 37 & $\ldots$ & $T$ & $T$ & 22 & 35,34 \\
\hline 26 & 34 & 14 & 24 & 41 & $\ldots$ & 0.00 & 0.0 & 28 & 29,31 \\
\hline 27 & 30 & 15 & 23 & 42 & $\ldots$ & 0.02 & 0.2 & 31 & 31 \\
\hline 28 & 29 & 9 & 19 & 46 & $\ldots$ & 0.00 & 0.0 & 25 & 35,01 \\
\hline 29 & 34 & 12 & 23 & 42 & $\ldots$ & 0.00 & 0.0 & 24 & 35 \\
\hline 30 & 42 & 7 & 25 & 40 & - & 0.00 & 0.0 & 14 & 28,30 \\
\hline 31 & 39 & 13 & 26 & 39 & - & 0.00 & 0.0 & 22 & 19 \\
\hline Total & $\ldots$ & $\ldots$ & $\ldots$ & 1,046 & $\ldots$ & 3.39 & 4.3 & $\ldots$ & $\ldots$ \\
\hline Mean & 37.9 & 24.1 & 31.3 & $\ldots$ & $\ldots$ & $\ldots$ & - & $\ldots$ & $\ldots$ \\
\hline Extreme & 64 & 7 & $\ldots$ & $\ldots$ & $\ldots$ & 0.64 & 1.2 & 39 & 29 \\
\hline
\end{tabular}




\begin{tabular}{|c|c|c|c|c|c|c|c|c|c|}
\hline \multicolumn{10}{|c|}{ Daily Climatological Data (Westover AFB, MA-February 1995) } \\
\hline \multirow[b]{3}{*}{ Day } & \multirow[b]{3}{*}{$\operatorname{Max}$} & \multirow[b]{3}{*}{ Min } & \multirow{3}{*}{$\begin{array}{l}\text { Temperatur } \\
\text { Mean (T) } \\
\end{array}$} & \multirow{2}{*}{\multicolumn{2}{|c|}{$\begin{array}{l}\text { Degree Days } \\
\left.\text { (Base } 65^{\circ} \mathrm{F}\right)\end{array}$}} & \multicolumn{2}{|c|}{ Precipitation (in.) } & \multicolumn{2}{|c|}{ Max Wind } \\
\hline & & & & & & \multirow{2}{*}{$\begin{array}{l}24 \mathrm{Hr} \\
\text { Water } \\
\text { Equivalent } \\
\end{array}$} & \multirow{2}{*}{$\begin{array}{l}24 \mathrm{Hr} \\
\text { Snowfall } \\
\end{array}$} & \multirow{2}{*}{$\begin{array}{l}\text { Speed } \\
\text { (kts) }\end{array}$} & \multirow[b]{2}{*}{ Direction } \\
\hline & & & & Heating & Cooling & & & & \\
\hline 1 & 45 & 32 & 39 & 26 & $\ldots$ & 0.00 & 0.0 & 20 & 19,20 \\
\hline 2 & 37 & 15 & 26 & 39 & $\ldots$ & 0.00 & 0.0 & 28 & 02 \\
\hline 3 & 30 & 8 & 19 & 46 & $\ldots$ & 0.00 & 0.0 & 27 & 01 \\
\hline 4 & 34 & 20 & 27 & 38 & $\ldots$ & 1.00 & 9.0 & 33 & 35 \\
\hline 5 & 25 & 3 & 14 & 51 & $\ldots$ & $T$ & $T$ & 45 & 29 \\
\hline 6 & 12 & -3 & 5 & 60 & $\ldots$ & 0.00 & 0.0 & 36 & 29 \\
\hline 7 & 21 & -6 & 8 & 57 & $\ldots$ & 0.00 & 0.0 & 22 & 28 \\
\hline 8 & 24 & $-8^{*}$ & 8 & 57 & $\ldots$ & 0.00 & 0.0 & 31 & 31,29 \\
\hline 9 & 30 & 12 & 21 & 44 & $\ldots$ & 0.00 & 0.0 & 24 & $27,29,30$ \\
\hline 10 & 37 & 18 & 28 & 37 & $\ldots$ & $T$ & $T$ & 21 & 18,21 \\
\hline 11 & 45 & 22 & 34 & 31 & -- & 0.00 & 0.0 & 26 & 33,25 \\
\hline 12 & 19 & 6 & 13 & 52 & - & 0.00 & 0.0 & 37 & 26 \\
\hline 13 & 25 & 4 & 15 & 50 & $\ldots$ & 0.00 & 0.0 & 26 & 28 \\
\hline 14 & 28 & 4 & 16 & 49 & $\ldots$ & 0.00 & 0.0 & 27 & 28 \\
\hline 15 & 35 & -4 & 16 & 49 & $\ldots$ & 0.26 & $T$ & 24 & 19,20 \\
\hline 16 & 43 & 29 & 36 & 29 & $\ldots$ & 0.03 & 0.0 & 30 & 29 \\
\hline 17 & 42 & 21 & 32 & 33 & $\ldots$ & 0.00 & 0.0 & 16 & 28 \\
\hline 18 & 49 & 15 & 32 & 33 & $\ldots$ & 0.00 & 0.0 & 13 & 20 \\
\hline 19 & 53 & 18 & 36 & 29 & $\ldots$ & 0.00 & 0.0 & 19 & 17,21 \\
\hline 20 & 48 & 25 & 37 & 28 & $\ldots$ & 0.00 & 0.0 & 22 & 04,06 \\
\hline 21 & 40 & 33 & 37 & 28 & $\ldots$ & 0.05 & $T$ & 20 & 01 \\
\hline 22 & 37 & 25 & 31 & 34 & $\ldots$ & 0.01 & $T$ & 21 & 34 \\
\hline 23 & 43 & 27 & 35 & 30 & - & 0.03 & 0.0 & 18 & 20 \\
\hline 24 & 39 & 23 & 31 & 34 & $\ldots$ & 0.45 & $T$ & 39 & 30 \\
\hline 25 & 35 & 17 & 26 & 39 & $\ldots$ & $T$ & $T$ & 38 & 29 \\
\hline 26 & 30 & 11 & 21 & 44 & - & 0.00 & 0.0 & 20 & 36 \\
\hline 27 & 26 & 13 & 20 & 45 & - & 0.03 & 0.3 & 15 & 36 \\
\hline 28 & 34 & 27 & 31 & 34 & $\ldots$ & 0.98 & 0.0 & 16 & 35 \\
\hline \multicolumn{10}{|l|}{29} \\
\hline \multicolumn{10}{|l|}{30} \\
\hline \multicolumn{10}{|l|}{31} \\
\hline Total & - & $\ldots$ & - & 1,126 & - & 2.84 & 9.3 & - & $\ldots$ \\
\hline Mean & 34.5 & 14.5 & 24.8 & - & - & $\ldots$ & $\ldots$ & - & $\ldots$ \\
\hline Extreme & 53 & -8 & $\ldots$ & - & - & 1.00 & 9.0 & 45 & 29 \\
\hline
\end{tabular}




\begin{tabular}{|c|c|c|c|c|c|c|c|c|c|}
\hline \multicolumn{10}{|c|}{ Daily Climatological Data (Westover AFB, MA-March 1995) } \\
\hline \multirow[b]{3}{*}{ Day } & \multirow[b]{3}{*}{ Max } & \multirow[b]{3}{*}{ Min } & \multicolumn{3}{|c|}{ Temperature (F) } & \multicolumn{2}{|c|}{ Precipitation (in.) } & \multicolumn{2}{|c|}{ Max Wind } \\
\hline & & & \multirow[b]{2}{*}{ Mean $(\tau)$} & \multicolumn{2}{|c|}{$\begin{array}{l}\text { Degree Days } \\
\left(\text { Base } 65^{\circ} \mathrm{F}\right)\end{array}$} & \multirow{2}{*}{$\begin{array}{l}24 \mathrm{Hr} \\
\text { Water } \\
\text { Equivalent } \\
\end{array}$} & \multirow{2}{*}{$\begin{array}{l}24 \mathrm{Hr} \\
\text { Snowfall } \\
\end{array}$} & \multirow{2}{*}{$\begin{array}{l}\text { Speed } \\
\text { (kts) }\end{array}$} & \multirow[b]{2}{*}{ Direction } \\
\hline & & & & Heating & Cooling & & & & \\
\hline 1 & 37 & 30 & 34 & 31 & 0 & 0.00 & 0 & 19 & 33 \\
\hline 2 & 35 & 23 & 29 & 36 & 0 & 0.00 & 0 & 25 & 35 \\
\hline 3 & 36 & 19 & 28 & 37 & 0 & 0.00 & 0 & 18 & 32 \\
\hline 4 & 40 & 21 & 31 & 34 & 0 & 0.00 & 0 & 13 & 23 \\
\hline 5 & 44 & 20 & 32 & 33 & 0 & 0.04 & 0 & 19 & 01,04 \\
\hline 6 & 45 & 34 & 40 & 25 & 0 & 0.01 & 0 & 12 & 35 \\
\hline 7 & 58 & 34 & 46 & 19 & 0 & 0.02 & 0 & 21 & 17 \\
\hline 8 & 61 & 38 & 50 & 15 & 0 & 0.60 & 0 & 34 & 17 \\
\hline 9 & 38 & 17 & 28 & 37 & 0 & 0.48 & 0 & 31 & 34 \\
\hline 10 & 34 & 13 & 29 & 36 & 0 & 0.00 & 0 & 27 & 33 \\
\hline 11 & 37 & 22 & 30 & 35 & 0 & 0.00 & 0 & 16 & 18 \\
\hline 12 & 47 & 32 & 35 & 30 & 0 & 0.00 & 0 & 21 & 22 \\
\hline 13 & 69 & 36 & 53 & 12 & 0 & 0.00 & 0 & 17 & 34 \\
\hline 14 & 53 & 34 & 44 & 21 & 0 & $T$ & 0 & 14 & 08 \\
\hline 15 & 63 & 42 & 53 & 12 & 0 & 0.01 & $\ldots$ & 11 & 05 \\
\hline 16 & 62 & 45 & 54 & 11 & 0 & $T$ & 0 & 18 & 16 \\
\hline 17 & 55 & 43 & 49 & 16 & 0 & 0.10 & 0 & 18 & 33,31 \\
\hline 18 & 53 & 36 & 45 & 20 & 0 & 0.01 & 0 & 25 & 35 \\
\hline 19 & 56 & 27 & 42 & 23 & 0 & 0.00 & 0 & 21 & 17 \\
\hline 20 & 53 & 31 & 42 & 23 & 0 & 0.01 & 0 & 27 & 22 \\
\hline 21 & 56 & 44 & 50 & 15 & 0 & 0.21 & 0 & 23 & 28,30 \\
\hline 22 & 50 & 41 & 46 & 19 & 0 & $T$ & 0 & 25 & 33,27 \\
\hline 23 & 51 & 38 & 45 & 20 & 0 & 0.00 & 0 & 25 & 35 \\
\hline 24 & 40 & 26 & 33 & 32 & 0 & $T$ & $\mathrm{~T}$ & 28 & 01,36 \\
\hline 25 & 49 & 26 & 38 & 27 & 0 & 0.00 & 0 & 34 & 01,36 \\
\hline 26 & 56 & 29 & 43 & 22 & 0 & 0.00 & 0 & 30 & 36 \\
\hline 27 & 55 & 26 & 41 & 24 & 0 & 0.00 & 0 & 25 & 02,34 \\
\hline 28 & 55 & 26 & 41 & 24 & 0 & 0.00 & 0 & 24 & 34,35 \\
\hline 29 & 60 & 23 & 42 & 23 & 0 & 0.00 & 0 & 22 & 17 \\
\hline 30 & 47 & 32 & 40 & 25 & 0 & 0.24 & 0 & 18 & 20 \\
\hline 31 & 50 & 34 & 42 & 23 & 0 & 0.06 & 0 & 28 & 29 \\
\hline Total & $\ldots$ & - & $\ldots$ & 760 & 0 & 1.79 & $T$ & $\ldots$ & $\ldots$ \\
\hline Mean & 49.8 & 30.4 & 40.5 & - & $\ldots$ & $\ldots$ & -- & $\ldots$ & $\ldots$ \\
\hline Extreme & 69 & 13 & -- & $\ldots$ & $\ldots$ & 0.60 & $T$ & 34 & $01,17,36$ \\
\hline
\end{tabular}




\begin{tabular}{|c|c|c|c|c|c|c|c|c|c|}
\hline \multicolumn{10}{|c|}{ Daily Climatological Data (Westover AFB, MA-April 1995) } \\
\hline \multirow[b]{3}{*}{ Day } & \multirow[b]{3}{*}{ Max } & \multirow[b]{3}{*}{ Min } & \multicolumn{3}{|c|}{ Temperature (F) } & \multicolumn{2}{|c|}{ Precipitation (in.) } & \multicolumn{2}{|c|}{ Max Wind } \\
\hline & & & \multirow[b]{2}{*}{ Mean $(T)$} & \multicolumn{2}{|c|}{$\begin{array}{l}\text { Degree Days } \\
\left(\text { Base } 65^{\circ} \mathrm{F}\right) \\
\end{array}$} & \multirow{2}{*}{$\begin{array}{l}24 \mathrm{Hr} \\
\text { Water } \\
\text { Equivalent } \\
\end{array}$} & \multirow{2}{*}{$\begin{array}{l}24 \mathrm{Hr} \\
\text { Snowfall } \\
\end{array}$} & \multirow{2}{*}{$\begin{array}{l}\text { Speed } \\
\text { (kts) }\end{array}$} & \multirow[b]{2}{*}{ Direction } \\
\hline & & & & Heating & Cooling & & & & \\
\hline 1 & 48 & 27 & 38 & 27 & $\ldots$ & 0.00 & 0.0 & 23 & 34 \\
\hline 2 & 48 & 24 & 36 & 29 & -- & 0.00 & 0.0 & 20 & 31 \\
\hline 3 & 51 & 18 & 35 & 30 & $\ldots$ & $T$ & 0.0 & 25 & 17,19 \\
\hline 4 & 60 & 22 & 41 & 24 & - & 0.23 & 0.0 & 49 & 25 \\
\hline 5 & 29 & $18^{*}$ & 24 & 41 & $\ldots$ & 0.00 & 0.0 & 44 & 27,28 \\
\hline 6 & 44 & 22 & 33 & 32 & $\ldots$ & 0.00 & 0.0 & 21 & 22 \\
\hline 7 & 55 & 29 & 41 & 24 & $\ldots$ & 0.00 & 0.0 & 23 & 35 \\
\hline 8 & 38 & $21^{*}$ & 30 & 35 & $\ldots$ & 0.04 & 0.4 & 17 & 20 \\
\hline 9 & 57 & 34 & 41 & 24 & -- & 0.12 & 0.0 & 24 & 36 \\
\hline 10 & 52 & 27 & 40 & 25 & - & 0.00 & 0.0 & 27 & 35 \\
\hline 11 & 61 & 24 & 43 & 22 & $\ldots$ & 0.00 & 0.0 & 20 & 18 \\
\hline 12 & 48 & 33 & 41 & 24 & $\ldots$ & 0.39 & 0.0 & 11 & 14 \\
\hline 13 & 60 & 45 & 53 & 12 & $\ldots$ & 0.70 & 0.0 & 30 & 30 \\
\hline 14 & 53 & 41 & 47 & 18 & $\ldots$ & 0.00 & 0.0 & 25 & 30 \\
\hline 15 & 51 & 38 & 45 & 20 & $\ldots$ & 0.00 & 0.0 & 32 & 34 \\
\hline 16 & 49 & 30 & 40 & 25 & $\ldots$ & 0.00 & 0.0 & 32 & 30 \\
\hline 17 & 62 & $24^{*}$ & 43 & 22 & $\ldots$ & $T$ & 0.0 & 20 & 04 \\
\hline 18 & 69 & 32 & 51 & 14 & $\ldots$ & 0.00 & 0.0 & 19 & 18 \\
\hline 19 & 59 & 46 & 53 & 12 & -- & 0.52 & 0.0 & 14 & 31 \\
\hline 20 & 68 & 49 & 59 & 6 & -- & 0.00 & 0.0 & 25 & 33 \\
\hline 21 & 58 & 45 & 52 & 13 & $\ldots$ & 0.30 & 0.0 & 14 & 14 \\
\hline 22 & 66 & 45 & 56 & 9 & -- & $\mathrm{T}$ & 0.0 & 35 & 29 \\
\hline 23 & 56 & 36 & 46 & 19 & $\ldots$ & 0.00 & 0.0 & 30 & 29 \\
\hline 24 & 64 & 32 & 48 & 17 & $\ldots$ & 0.00 & 0.0 & 17 & 33 \\
\hline 25 & 65 & 33 & 49 & 16 & $\ldots$ & 0.00 & 0.0 & 20 & $01,35,36$ \\
\hline 26 & 61 & 40 & 51 & 14 & $\ldots$ & 0.00 & 0.0 & 26 & 25 \\
\hline 27 & 74 & 37 & 59 & 6 & $\ldots$ & $T$ & 0.0 & 21 & 19,22 \\
\hline 28 & 63 & 49 & 56 & 9 & $\ldots$ & 0.09 & 0.0 & 16 & 35 \\
\hline 29 & 62 & 44 & 53 & 12 & $\ldots$ & $T$ & 0.0 & 17 & 29 \\
\hline 30 & 60 & 44 & 52 & 13 & - & 0.11 & 0.0 & 17 & 09 \\
\hline \multicolumn{10}{|l|}{31} \\
\hline Total & - & $\ldots$ & $\ldots$ & 594 & 0 & 2.50 & 0.4 & - & - \\
\hline Mean & 56.4 & 33.6 & 45.2 & $\ldots$ & $\ldots$ & $\ldots$ & --- & - & $\ldots$ \\
\hline Extreme & 74 & 18 & $\ldots$ & - & -- & 0.70 & 0.4 & 49 & 25 \\
\hline
\end{tabular}




\begin{tabular}{|c|c|c|c|c|c|c|c|c|c|}
\hline \multicolumn{10}{|c|}{ Daily Climatological Data (Westover AFB, MA-May 1995) } \\
\hline \multirow[b]{3}{*}{ Day } & \multirow[b]{3}{*}{ Max } & \multirow[b]{3}{*}{ Min } & \multicolumn{3}{|c|}{ Temperature (F) } & \multicolumn{2}{|c|}{ Precipitation (in.) } & \multicolumn{2}{|c|}{ Max Wind } \\
\hline & & & \multirow[b]{2}{*}{ Mean $(T)$} & \multicolumn{2}{|c|}{$\begin{array}{l}\text { Degree Days } \\
\text { (Base } 65^{\circ} \mathrm{F} \text { ) }\end{array}$} & \multirow{2}{*}{$\begin{array}{l}24 \mathrm{Hr} \\
\text { Water } \\
\text { Equivalent } \\
\end{array}$} & \multirow{2}{*}{$\begin{array}{l}24 \mathrm{Hr} \\
\text { Snowfall } \\
\end{array}$} & \multirow{2}{*}{$\begin{array}{l}\text { Speed } \\
\text { (kts) }\end{array}$} & \multirow[b]{2}{*}{ Direction } \\
\hline & & & & Heating & Cooling & & & & \\
\hline 1 & 63 & 37 & 50 & 15 & 0 & 0.00 & 0 & 24 & 02 \\
\hline 2 & 61 & 32 & 47 & 18 & 0 & 0.00 & 0 & 14 & 36 \\
\hline 3 & 68 & 40 & 54 & 11 & 0 & 0.00 & 0 & 25 & 36 \\
\hline 4 & 73 & 35 & 54 & 11 & 0 & 0.00 & 0 & 16 & 23 \\
\hline 5 & 57 & 45 & 51 & 14 & 0 & 0.05 & 0 & 15 & 33 \\
\hline 6 & 64 & 38 & 51 & 14 & 0 & 0.00 & 0 & 32 & 30,33 \\
\hline 7 & 62 & 27 & 45 & 20 & 0 & 0.00 & 0 & 36 & 35 \\
\hline 8 & 68 & 42 & 55 & 10 & 0 & 0.00 & 0 & 42 & 36 \\
\hline 9 & 75 & 34 & 55 & 10 & 0 & 0.00 & 0 & 21 & 35 \\
\hline 10 & 56 & 49 & 53 & 12 & 0 & 0.10 & 0 & 24 & 20 \\
\hline 11 & 51 & 48 & 50 & 15 & 0 & 0.48 & 0 & 17 & 36 \\
\hline 12 & 67 & 47 & 57 & 8 & 0 & 0.12 & 0 & 14 & 02 \\
\hline 13 & 62 & 43 & 53 & 12 & 0 & 0.03 & 0 & 23 & $04,07,36$ \\
\hline 14 & 75 & 37 & 56 & 9 & 0 & 0.01 & 0 & 23 & 13 \\
\hline 15 & 56 & 43 & 50 & 15 & 0 & 0.34 & 0 & 13 & 18 \\
\hline 16 & 77 & 36 & 57 & 8 & 0 & 0.00 & 0 & 29 & 28 \\
\hline 17 & 62 & 49 & 56 & 9 & 0 & 0.10 & 0 & 15 & 16 \\
\hline 18 & 67 & 54 & 61 & 4 & 0 & $T$ & 0 & 19 & 34 \\
\hline 19 & 58 & 48 & 53 & 12 & 0 & 0.07 & 0 & 13 & 34 \\
\hline 20 & 73 & 40 & 57 & 8 & 0 & 0.00 & 0 & 25 & 33 \\
\hline 21 & 81 & 39 & 60 & 5 & 0 & $T$ & 0 & 28 & 18 \\
\hline 22 & 71 & 47 & 59 & 6 & 0 & 0.00 & 0 & 27 & 27 \\
\hline 23 & 79 & 39 & 59 & 6 & 0 & 0.00 & 0 & 26 & 23 \\
\hline 24 & 87 & 58 & 73 & 0 & 8 & 0.66 & 0 & 26 & 22 \\
\hline 25 & 68 & 57 & 63 & 2 & 0 & 0.12 & 0 & 21 & 35 \\
\hline 26 & 69 & 53 & 61 & 4 & 0 & $T$ & 0 & 14 & 31 \\
\hline 27 & 76 & 46 & 61 & 4 & 0 & 0.00 & 0 & 18 & 02,36 \\
\hline 28 & 72 & 41 & 57 & 8 & 0 & 0.02 & 0 & 22 & 21 \\
\hline 29 & 70 & 54 & 62 & 3 & 0 & 0.77 & 0 & 26 & 20 \\
\hline 30 & 74 & 50 & 62 & 3 & 0 & $T$ & 0 & 25 & 34 \\
\hline 31 & 87 & 48 & 68 & 0 & 3 & 0.00 & 0 & 23 & $30 / 28$ \\
\hline Total & $\ldots$ & $\ldots$ & $\ldots$ & 276 & 11 & 2.87 & 0 & $\ldots$ & $\ldots$ \\
\hline Mean & 68.7 & 43.7 & 56.5 & $\ldots$ & $\ldots$ & $\ldots$ & $\ldots$ & $\ldots$ & $\ldots$ \\
\hline Extreme & 87 & 27 & $\ldots$ & $\ldots$ & - & 0.77 & 0 & 42 & 36 \\
\hline
\end{tabular}




\begin{tabular}{|c|c|c|c|c|c|c|c|c|c|}
\hline \multicolumn{10}{|c|}{ Daily Climatological Data (Westover AFB, MA-June 1995) } \\
\hline \multirow[b]{3}{*}{ Day } & \multicolumn{5}{|c|}{ Temperature (F) } & \multicolumn{2}{|c|}{ Precipitation (in.) } & \multicolumn{2}{|c|}{ Max Wind } \\
\hline & \multirow[b]{2}{*}{ Max } & \multirow[b]{2}{*}{ Min } & \multirow[b]{2}{*}{ Mean $(T)$} & \multicolumn{2}{|c|}{$\begin{array}{l}\text { Degree Days } \\
\text { (Base } 65^{\circ} \mathrm{F} \text { ) }\end{array}$} & \multirow{2}{*}{$\begin{array}{l}24 \mathrm{Hr} \\
\text { Water } \\
\text { Equivalent } \\
\end{array}$} & \multirow{2}{*}{$\begin{array}{l}24 \mathrm{Hr} \\
\text { Snowfall } \\
\end{array}$} & \multirow{2}{*}{$\begin{array}{l}\text { Speed } \\
\text { (kts) }\end{array}$} & \multirow[b]{2}{*}{ Direction } \\
\hline & & & & Heating & Cooling & & & & \\
\hline 1 & 87 & 51 & 69 & -- & 4 & 0.00 & 0 & 23 & 24 \\
\hline 2 & 80 & 59 & 70 & $\ldots$ & 5 & $T$ & 0 & 21 & 17 \\
\hline 3 & 83 & 64 & 74 & $\ldots$ & 9 & 0.07 & 0 & $M$ & $\mathrm{M}$ \\
\hline 4 & 79 & 49 & 64 & 1 & $\ldots$ & 0.39 & 0 & 23 & 35 \\
\hline 5 & 83 & 45 & 64 & 1 & - & 0.00 & 0 & 20 & 25 \\
\hline 6 & 84 & 51 & 68 & $\ldots$ & 3 & 0.00 & 0 & 18 & 21 \\
\hline 7 & 77 & 62 & 70 & -- & 5 & 0.07 & 0 & 19 & 35,36 \\
\hline 8 & 80 & 55 & 68 & $\ldots$ & 3 & $T$ & 0 & 19 & 35,36 \\
\hline 9 & 77 & 48 & 63 & 2 & $\ldots$ & 0.00 & 0 & 17 & 35 \\
\hline 10 & 79 & 50 & 65 & -- & $\ldots$ & 0.00 & 0 & 17 & 19 \\
\hline 11 & 70 & 55 & 63 & 2 & $\ldots$ & 0.15 & 0 & 15 & 16 \\
\hline 12 & 67 & 56 & 62 & 3 & - & 0.19 & 0 & 15 & 34 \\
\hline 13 & 70 & 56 & 63 & 2 &.- & 0.02 & 0 & 13 & 32,34 \\
\hline 14 & 68 & 51 & 60 & 5 & $\ldots$ & 0.02 & 0 & 17 & 02,34 \\
\hline 15 & 73 & 46 & 60 & 5 & $\ldots$ & $T$ & 0 & 22 & 01,36 \\
\hline 16 & 84 & 44 & 64 & 1 & $\ldots$ & 0.00 & 0 & 17 & 27 \\
\hline 17 & 88 & 48 & 68 & -- & 3 & 0.00 & 0 & 18 & 28 \\
\hline 18 & 92 & 54 & 73 & $\ldots$ & 8 & 0.00 & 0 & 22 & 27 \\
\hline 19 & $97^{*}$ & 65 & 81 & $\ldots$ & 16 & 0.00 & 0 & 22 & 24 \\
\hline 20 & 93 & 65 & 79 & - & 14 & 0.37 & 0 & 21 & 02 \\
\hline 21 & 82 & 52 & 67 & $\ldots$ & 2 & 0.00 & 0 & 16 & 20 \\
\hline 22 & 80 & 55 & 68 & - & 3 & 0.00 & 0 & 14 & $19,20,17$ \\
\hline 23 & 82 & 50 & 66 & $\ldots$ & 1 & 0.00 & 0 & 12 & 06 \\
\hline 24 & 82 & 51 & 67 & - & 2 & 0.00 & 0 & 14 & 27 \\
\hline 25 & 86 & 66 & 76 & -- & 11 & 0.02 & 0 & 14 & 28 \\
\hline 26 & 84 & 68 & 76 & $\ldots$ & 11 & 0.06 & 0 & 24 & 07 \\
\hline 27 & 76 & 48 & 62 & 3 & $\ldots$ & 0.00 & 0 & 22 & 05 \\
\hline 28 & 79 & $39 *$ & 59 & 6 & $\ldots$ & 0.00 & 0 & 12 & 19 \\
\hline 29 & 86 & 48 & 67 & $\ldots$ & 2 & 0.00 & 0 & 14 & 19 \\
\hline 30 & 89 & 57 & 73 & $\ldots$ & 8 & 0.00 & 0 & 18 & 20 \\
\hline 31 & $\ldots$ & - & $\ldots$ & $\ldots$ & - & $\ldots$ & $\ldots$ & $\ldots$ & $\ldots$ \\
\hline Total & $\ldots$ & - & $\ldots$ & 31 & 110 & 1.36 & 0 & $\ldots$ & $\ldots$ \\
\hline Mean & 81.2 & 53.6 & 67.6 & $\ldots$ & - & $\ldots$ & $\ldots$ & - & $\ldots$ \\
\hline Extreme & 97 & 39 & -- & - & $\ldots$ & 0.39 & 0 & 24 & 07 \\
\hline
\end{tabular}




\begin{tabular}{|c|c|c|c|c|c|c|c|c|c|}
\hline \multicolumn{10}{|c|}{ Daily Climatological Data (Westover AFB, MA-July 1995) } \\
\hline \multirow[b]{3}{*}{ Day } & \multirow[b]{3}{*}{ Max } & \multirow[b]{3}{*}{ Min } & \multicolumn{3}{|c|}{ Temperature (F) } & \multicolumn{2}{|c|}{ Precipitation (in.) } & \multicolumn{2}{|c|}{ Max Wind } \\
\hline & & & \multirow[b]{2}{*}{ Mean $(T)$} & \multicolumn{2}{|c|}{$\begin{array}{l}\text { Degree Days } \\
\left(\text { Base } 65^{\circ} \mathrm{F}\right)\end{array}$} & \multirow{2}{*}{$\begin{array}{l}24 \mathrm{Hr} \\
\text { Water } \\
\text { Equivalent } \\
\end{array}$} & \multirow{2}{*}{$\begin{array}{l}24 \mathrm{Hr} \\
\text { Snowfall } \\
\end{array}$} & \multirow{2}{*}{$\begin{array}{l}\text { Speed } \\
\text { (kts) } \\
\end{array}$} & \multirow[b]{2}{*}{ Direction } \\
\hline & & & & Heating & Cooling & & & & \\
\hline 1 & 82 & 65 & 74 & -- & 9 & $2.05^{*}$ & 0 & 45 & 23 \\
\hline 2 & 80 & 56 & 68 & -- & 3 & 0.00 & 0 & 21 & 28 \\
\hline 3 & 81 & 48 & 65 & $\ldots$ & $\ldots$ & 0.00 & 0 & 19 & 35,34 \\
\hline 4 & 84 & 51 & 63 & $\ldots$ & 2 & 0.00 & 0 & 16 & 18,19 \\
\hline 5 & 83 & 59 & 71 & $\ldots$ & 6 & 0.00 & 0 & 18 & 18,19 \\
\hline 6 & 86 & 67 & 77 & - & 12 & 0.00 & 0 & 20 & 21,22 \\
\hline 7 & 82 & 69 & 76 & - & 11 & $\mathrm{~T}$ & 0 & 19 & $26,20,21$ \\
\hline 8 & 83 & 61 & 72 & -- & 7 & 0.30 & 0 & 38 & 26 \\
\hline 9 & 75 & 53 & 64 & 1 & $\ldots$ & 0.00 & 0 & 19 & 27,29 \\
\hline 10 & 83 & 55 & 69 & - & 4 & 0.00 & 0 & 19 & 25 \\
\hline 11 & 80 & 61 & 71 & - & 6 & 0.30 & 0 & 15 & 05 \\
\hline 12 & 83 & 62 & 73 & $\ldots$ & 8 & 0.00 & 0 & 14 & 19,20 \\
\hline 13 & 90 & 64 & 77 & $\ldots$ & 12 & 0.00 & 0 & 19 & 23,24 \\
\hline 14 & $99 *$ & 67 & 83 & $\ldots$ & 18 & 0.00 & 0 & 19 & 25,26 \\
\hline 15 & $96^{*}$ & 69 & 83 & $\ldots$ & 18 & 0.05 & 0 & 35 & 34 \\
\hline 16 & 85 & 63 & 74 & $\ldots$ & 9 & $\mathrm{~T}$ & 0 & 17 & 20 \\
\hline 17 & 75 & 62 & 69 & -- & 4 & 0.24 & 0 & 13 & 09 \\
\hline 18 & 81 & 61 & 71 & -- & 6 & 0.15 & 0 & 16 & 04 \\
\hline 19 & 85 & 60 & 73 & -- & 8 & 0.00 & 0 & 17 & 27 \\
\hline 20 & 88 & 58 & 73 & $\ldots$ & 8 & 0.00 & 0 & 24 & 23 \\
\hline 21 & 83 & 64 & 74 & $\ldots$ & 9 & 0.02 & 0 & 14 & 23 \\
\hline 22 & 90 & 61 & 76 & $\ldots$ & 11 & 0.00 & 0 & 13 & 11,15 \\
\hline 23 & 85 & 69 & 77 & -- & 12 & 0.03 & 0 & 17 & 17,20 \\
\hline 24 & 91 & 70 & 81 & $\ldots$ & 16 & 0.17 & 0 & 15 & $23,19,24$ \\
\hline 25 & 88 & 70 & 79 & -- & 14 & 0.01 & 0 & 14 & 30 \\
\hline 26 & 91 & 72 & 82 & $\ldots$ & 17 & 0.04 & 0 & 25 & 24 \\
\hline 27 & $94^{*}$ & 69 & 82 & $\ldots$ & 17 & $T$ & 0 & 17 & 30 \\
\hline 28 & 91 & 70 & 81 & - & 16 & $T$ & 0 & 18 & 19,17 \\
\hline 29 & 93 & 70 & 82 & $\ldots$ & 17 & 0.54 & 0 & 25 & 20 \\
\hline 30 & 90 & 61 & 76 & - & 11 & 0.00 & 0 & 19 & 33 \\
\hline 31 & 92 & 56 & 74 & - & 9 & 0.00 & 0 & 15 & 23 \\
\hline Total & $\ldots$ & - & $\ldots$ & 1 & 300 & 3.90 & 0 & $\ldots$ & $\ldots$ \\
\hline Mean & 86.1 & 62.7 & 74.5 & $\ldots$ & - & $\ldots$ & $\ldots$ & $\ldots$ & $\ldots$ \\
\hline Extreme & 99 & 48 & $\ldots$ & $\ldots$ & $\ldots$ & 2.05 & 0 & 38 & 26 \\
\hline
\end{tabular}




\begin{tabular}{|c|c|c|c|c|c|c|c|c|c|}
\hline \multicolumn{10}{|c|}{ Daily Climatological Data (Westover AFB, MA-August 1995) } \\
\hline \multirow[b]{3}{*}{ Day } & \multirow[b]{3}{*}{ Max } & \multirow[b]{3}{*}{ Min } & \multicolumn{3}{|c|}{ Temperature (F) } & \multicolumn{2}{|c|}{ Precipitation (in.) } & \multicolumn{2}{|c|}{ Max Wind } \\
\hline & & & \multirow[b]{2}{*}{ Mean (T) } & \multicolumn{2}{|c|}{$\begin{array}{l}\text { Degree Days } \\
\left(\text { Base } 65^{\circ} \mathrm{F}\right)\end{array}$} & \multirow{2}{*}{$\begin{array}{l}24 \mathrm{Hr} \\
\text { Water } \\
\text { Equivalent } \\
\end{array}$} & \multirow{2}{*}{$\begin{array}{l}24 \mathrm{Hr} \\
\text { Snowfall }\end{array}$} & \multirow{2}{*}{$\begin{array}{l}\text { Speed } \\
\text { (kts) }\end{array}$} & \multirow[b]{2}{*}{ Direction } \\
\hline & & & & Heating & Cooling & & & & \\
\hline 1 & $96^{*}$ & 61 & 79 & 0 & 14 & 0.00 & 0 & 21 & 22 \\
\hline 2 & 94 & 65 & 80 & 0 & 15 & $1.64^{*}$ & 0 & 31 & 23 \\
\hline 3 & 76 & 69 & 73 & 0 & 8 & $T$ & 0 & 10 & 06,07 \\
\hline 4 & 93 & 68 & 81 & 0 & 16 & 0.28 & 0 & 32 & 32 \\
\hline 5 & 78 & 65 & 72 & 0 & 7 & 0.95 & 0 & 14 & 08,07 \\
\hline 6 & 69 & 62 & 66 & 0 & 1 & 0.64 & 0 & 21 & 05 \\
\hline 7 & 78 & 51 & 65 & 0 & 0 & 0.00 & 0 & 20 & 02,03 \\
\hline 8 & 82 & $45^{*}$ & 64 & 1 & 0 & 0.00 & 0 & 14 & 31 \\
\hline 9 & 86 & 52 & 69 & 0 & 4 & 0.00 & 0 & 13 & 20 \\
\hline 10 & 86 & 52 & 69 & 0 & 4 & 0.00 & 0 & 17 & 20 \\
\hline 11 & 88 & 63 & 71 & 0 & 6 & 0.00 & 0 & 23 & 23 \\
\hline 12 & 85 & 70 & 78 & 0 & 13 & 0.01 & 0 & 18 & 28 \\
\hline 13 & 86 & 60 & 73 & 0 & 8 & 0.00 & 0 & 17 & 32 \\
\hline 14 & 87 & 56 & 72 & 0 & 7 & 0.00 & 0 & 14 & 18 \\
\hline 15 & 86 & 68 & 77 & 0 & 12 & $T$ & 0 & 17 & 16 \\
\hline 16 & 91 & 64 & 78 & 0 & 13 & 0.00 & 0 & 14 & 19 \\
\hline 17 & 92 & 68 & 80 & 0 & 15 & 0.00 & 0 & 16 & 02,04 \\
\hline 18 & 86 & 60 & 73 & 0 & 8 & 0.00 & 0 & 21 & 05 \\
\hline 19 & 82 & 50 & 66 & 0 & 1 & 0.00 & 0 & 20 & 07 \\
\hline 20 & 84 & $40^{*}$ & 62 & 3 & 0 & 0.00 & 0 & 12 & 35,04 \\
\hline 21 & 94 & 47 & 71 & 0 & 6 & 0.00 & 0 & 20 & 26 \\
\hline 22 & 81 & 54 & 68 & 0 & 3 & 0.00 & 0 & 23 & 35 \\
\hline 23 & 80 & 48 & 64 & 1 & 0 & 0.00 & 0 & 17 & 27 \\
\hline 24 & 84 & 59 & 72 & 0 & 7 & 0.00 & 0 & 32 & 28 \\
\hline 25 & 74 & 50 & 62 & 3 & 0 & 0.00 & 0 & 25 & 01 \\
\hline 26 & 72 & 42 & 57 & 8 & 0 & 0.00 & 0 & 11 & 17 \\
\hline 27 & 73 & 54 & 64 & 1 & 0 & 0.01 & 0 & 19 & 36 \\
\hline 28 & 82 & 47 & 65 & 0 & 0 & 0.00 & 0 & 15 & 07 \\
\hline 29 & 83 & 44 & 64 & 1 & 0 & 0.00 & 0 & 17 & 01 \\
\hline 30 & 82 & 47 & 65 & 0 & 0 & 0.00 & 0 & 24 & 35 \\
\hline 31 & 89 & 43 & 66 & 0 & 1 & 0.02 & 0 & 23 & 22 \\
\hline Total & $\ldots$ & $\ldots$ & $\ldots$ & 18 & 169 & 3.55 & 0 & $\ldots$ & $\ldots$ \\
\hline Mean & 83.8 & 55.6 & 69.9 & $\ldots$ & $\ldots$ & $\ldots$ & $\ldots$ & $\ldots$ & $\ldots$ \\
\hline Extreme & 96 & 40 & - & $\ldots$ & $\ldots$ & 1.64 & 0 & 32 & 32,28 \\
\hline
\end{tabular}




\begin{tabular}{|c|c|c|c|c|c|c|c|c|c|}
\hline \multicolumn{10}{|c|}{ Daily Climatological Data (Westover AFB, MA-September 1995) } \\
\hline \multirow[b]{3}{*}{ Day } & \multirow[b]{3}{*}{ Max } & \multirow[b]{3}{*}{ Min } & \multicolumn{3}{|c|}{ Temperature (F) } & \multicolumn{2}{|c|}{ Precipitation (in.) } & \multicolumn{2}{|c|}{ Max Wind } \\
\hline & & & \multirow[b]{2}{*}{ Mean $(\mathrm{T})$} & \multicolumn{2}{|c|}{$\begin{array}{l}\text { Degree Days } \\
\left(\text { Base } 65^{\circ} \mathrm{F}\right)\end{array}$} & \multirow{2}{*}{$\begin{array}{l}24 \mathrm{Hr} \\
\text { Water } \\
\text { Equivalent } \\
\end{array}$} & \multirow{2}{*}{$\begin{array}{l}24 \mathrm{Hr} \\
\text { Snowfall } \\
\end{array}$} & \multirow{2}{*}{$\begin{array}{l}\begin{array}{l}\text { Speed } \\
\text { (kts) }\end{array} \\
\end{array}$} & \multirow[b]{2}{*}{ Directior } \\
\hline & & & & Heating & Cooling & & & & \\
\hline 1 & 84 & 64 & 74 & 0 & 9 & 0.00 & 0 & 20 & 29,01 \\
\hline 2 & 78 & 49 & 64 & 1 & 0 & 0.00 & 0 & 20 & 01 \\
\hline 3 & 77 & 42 & 60 & 5 & 0 & 0.00 & 0 & 16 & 36 \\
\hline 4 & 84 & 44 & 64 & 1 & 0 & 0.00 & 0 & 12 & 16,26 \\
\hline 5 & 90 & 57 & 74 & 0 & 9 & 0.00 & 0 & 21 & 27 \\
\hline 6 & 84 & 54 & 69 & 0 & 4 & 0.00 & 0 & 17 & 33 \\
\hline 7 & 90 & 65 & 78 & 0 & 13 & 0.00 & 0 & 24 & 24 \\
\hline 8 & 70 & 55 & 63 & 2 & 0 & 0.02 & 0 & 20 & 34 \\
\hline 9 & 65 & 55 & 60 & 5 & 0 & 0.01 & 0 & 14 & 30 \\
\hline 10 & 70 & 38 & 54 & 11 & 0 & 0.00 & 0 & 24 & 31 \\
\hline 11 & 74 & $32 *$ & 53 & 12 & 0 & 0.00 & 0 & 16 & 23 \\
\hline 12 & 79 & 38 & 59 & 6 & 0 & 0.00 & 0 & 21 & 19,21 \\
\hline 13 & 72 & 62 & 67 & 0 & 2 & 0.15 & 0 & 16 & 20 \\
\hline 14 & 88 & 57 & 73 & 0 & 8 & 0.04 & 0 & 31 & 29 \\
\hline 15 & 69 & 41 & 55 & 10 & 0 & 0.00 & 0 & 35 & 22 \\
\hline 16 & 69 & 36 & 53 & 12 & 0 & 0.00 & 0 & 18 & 18,20 \\
\hline 17 & 63 & 54 & 59 & 6 & 0 & 1.42 & 0 & 20 & 35 \\
\hline 18 & 71 & 42 & 57 & 8 & 0 & 0.00 & 0 & 19 & 35 \\
\hline 19 & 72 & 38 & 55 & 10 & 0 & 0.00 & 0 & 15 & 02 \\
\hline 20 & 71 & 40 & 56 & 9 & 0 & 0.00 & 0 & 17 & 26 \\
\hline 21 & 70 & 54 & 62 & 3 & 0 & $T$ & 0 & 12 & 19 \\
\hline 22 & 73 & 55 & 64 & 1 & 0 & $1.40^{*}$ & 0 & 28 & 28 \\
\hline 23 & 61 & 41 & 51 & 14 & 0 & 0.00 & 0 & 22 & 35 \\
\hline 24 & 64 & 35 & 50 & 15 & 0 & 0.00 & 0 & 12 & 03 \\
\hline 25 & 64 & 48 & 56 & 9 & 0 & 0.01 & 0 & 13 & 05,06 \\
\hline 26 & 60 & 54 & 57 & 8 & 0 & 0.78 & 0 & 12 & 33,30 \\
\hline 27 & 76 & 52 & 64 & 1 & 0 & 0.00 & 0 & 20 & 21,25 \\
\hline 28 & 70 & 37 & 54 & 11 & 0 & 0.00 & 0 & 22 & 36 \\
\hline 29 & 67 & 30 & 54 & 11 & 0 & 0.00 & 0 & 15 & 34 \\
\hline 30 & 70 & 35 & 53 & 12 & 0 & 0.00 & 0 & 13 & 29 \\
\hline \multicolumn{10}{|l|}{31} \\
\hline Total & $\ldots$ & $\ldots$ & $\ldots$ & 183 & 45 & 3.83 & 0 & $\ldots$ & $\ldots$ \\
\hline Mean & 73.2 & 46.8 & 60.4 & $\ldots$ & $\ldots$ & -- & - & $\ldots$ & - \\
\hline Extreme & 90 & 30 & -- & $\ldots$ & - & 1.42 & 0 & 35 & 22 \\
\hline
\end{tabular}




\begin{tabular}{|c|c|c|c|c|c|c|c|c|c|}
\hline \multicolumn{10}{|c|}{ Daily Climatological Data (Westover AFB, MA-October 1995) } \\
\hline \multirow[b]{3}{*}{ Day } & \multirow[b]{3}{*}{ Max } & \multirow[b]{3}{*}{ Min } & \multicolumn{3}{|c|}{ Temperature (F) } & \multicolumn{2}{|c|}{ Precipitation (in.) } & \multicolumn{2}{|c|}{ Max Wind } \\
\hline & & & \multirow[b]{2}{*}{ Mean $(T)$} & \multicolumn{2}{|c|}{$\begin{array}{l}\text { Degree Days } \\
\left.\text { (Base } 65^{\circ} \mathrm{F}\right)\end{array}$} & \multirow{2}{*}{$\begin{array}{l}24 \mathrm{Hr} \\
\text { Water } \\
\text { Equivalent } \\
\end{array}$} & \multirow{2}{*}{$\begin{array}{l}24 \mathrm{Hr} \\
\text { Snowfall } \\
\end{array}$} & \multirow{2}{*}{$\begin{array}{l}\text { Speed } \\
\text { (kts) }\end{array}$} & \multirow[b]{2}{*}{ Direction } \\
\hline & & & & Heating & Cooling & & & & \\
\hline 1 & 71 & 33 & 52 & 13 & 0 & 0.00 & 0 & 10 & 16,31 \\
\hline 2 & 81 & 35 & 58 & 7 & 0 & 0.00 & 0 & 13 & 27,25 \\
\hline 3 & 78 & 42 & 60 & 5 & 0 & 0.00 & 0 & 18 & 25,24 \\
\hline 4 & 69 & 57 & 63 & 2 & 0 & 0.24 & 0 & 16 & 20 \\
\hline 5 & 68 & 55 & 62 & 3 & 0 & $1.96^{*}$ & 0 & 18 & 06 \\
\hline 6 & 57 & 53 & 55 & 10 & 0 & 1.12 & 0 & 21 & 05 \\
\hline 7 & 57 & 52 & 55 & 10 & 0 & 0.02 & 0 & 15 & 33 \\
\hline 8 & 65 & 47 & 56 & 9 & 0 & $T$ & 0 & 21 & 28 \\
\hline 9 & 67 & 35 & 51 & 14 & 0 & 0.00 & 0 & 15 & 27 \\
\hline 10 & 69 & 34 & 52 & 13 & 0 & 0.00 & 0 & 11 & 17 \\
\hline 11 & 75 & 44 & 60 & 5 & 0 & 0.00 & 0 & 17 & 23 \\
\hline 12 & 81 & 43 & 62 & 3 & 0 & 0.00 & 0 & 21 & 27 \\
\hline 13 & 84 & 43 & 64 & 1 & 0 & 0.00 & 0 & 14 & 19 \\
\hline 14 & 71 & 44 & 58 & 7 & 0 & $2.08^{*}$ & 0 & 32 & 27 \\
\hline 15 & 61 & 45 & 53 & 12 & 0 & 0.35 & 0 & 23 & 26 \\
\hline 16 & 57 & 40 & 49 & 16 & 0 & $T$ & 0 & 30 & 26 \\
\hline 17 & 57 & 33 & 45 & 20 & 0 & $T$ & 0 & 22 & 29,32 \\
\hline 18 & 67 & 30 & 49 & 16 & 0 & 0.00 & 0 & 26 & 21 \\
\hline 19 & 71 & 38 & 55 & 10 & 0 & 0.00 & 0 & 14 & 28 \\
\hline 20 & 66 & 46 & 56 & 9 & 0 & 0.08 & 0 & 16 & 12,10 \\
\hline 21 & 71 & 52 & 62 & 3 & 0 & $1.84^{*}$ & 0 & 25 & 16 \\
\hline 22 & 61 & 40 & 51 & 14 & 0 & 0.00 & 0 & 25 & 24 \\
\hline 23 & 75 & 33 & 54 & 11 & 0 & 0.00 & 0 & 14 & 24 \\
\hline 24 & 71 & 37 & 54 & 11 & 0 & $T$ & 0 & 23 & 17,19 \\
\hline 25 & 61 & 35 & 48 & 17 & 0 & $T$ & 0 & 27 & 31 \\
\hline 26 & 59 & 31 & 45 & 20 & 0 & 0.00 & 0 & 13 & 18 \\
\hline 27 & 65 & 31 & 48 & 17 & 0 & 0.37 & 0 & 21 & 17 \\
\hline 28 & 71 & 49 & 60 & 5 & 0 & $2.17^{*}$ & 0 & 67 & 24 \\
\hline 29 & 54 & 41 & 48 & 17 & 0 & 0.00 & 0 & 31 & 28 \\
\hline 30 & 53 & 36 & 45 & 20 & 0 & 0.00 & 0 & 22 & 27 \\
\hline 31 & 44 & 34 & 39 & 26 & 0 & $T$ & 0 & 12 & 27 \\
\hline Total & $\ldots$ & $\ldots$ & $\ldots$ & 346 & 0 & $10.23^{*}$ & 0 & $\ldots$ & $\ldots$ \\
\hline Mean & 66.4 & 40.9 & 53.8 & $\ldots$ & $\ldots$ & $\ldots$ & $\ldots$ & - & -- \\
\hline Extreme & 84 & 30 & $\ldots$ & $\ldots$ & -- & 2.17 & 0 & 67 & 24 \\
\hline
\end{tabular}




\begin{tabular}{|c|c|c|c|c|c|c|c|c|c|}
\hline \multicolumn{10}{|c|}{ Daily Climatological Data (Westover AFB, MA-November 1995) } \\
\hline \multirow[b]{3}{*}{ Day } & \multirow[b]{3}{*}{ Max } & \multirow[b]{3}{*}{ Min } & \multicolumn{3}{|c|}{ Temperature (F) } & \multicolumn{2}{|c|}{ Precipitation (in.) } & \multicolumn{2}{|c|}{ Max Wind } \\
\hline & & & \multirow[b]{2}{*}{ Mean $(T)$} & \multicolumn{2}{|c|}{$\begin{array}{l}\text { Degree Days } \\
\text { (Base } 65^{\circ} \mathrm{F} \text { ) }\end{array}$} & \multirow{2}{*}{$\begin{array}{l}24 \mathrm{Hr} \\
\text { Water } \\
\text { Equivalent } \\
\end{array}$} & \multirow{2}{*}{$\begin{array}{l}24 \mathrm{Hi} \\
\text { Snowfall }\end{array}$} & \multirow{2}{*}{$\begin{array}{l}\text { Speed } \\
\text { (kts) }\end{array}$} & \multirow[b]{2}{*}{ Direction } \\
\hline & & & & Heating & Cooling & & & & \\
\hline 1 & 46 & 42 & 44 & 21 & 0 & 0.05 & 0.0 & 14 & 36 \\
\hline 2 & 48 & 42 & 45 & 20 & 0 & 0.65 & 0.0 & 16 & 33 \\
\hline 3 & 55 & 47 & 51 & 14 & 0 & 0.05 & 0.0 & 16 & 32 \\
\hline 4 & 46 & 36 & 41 & 24 & 0 & $T$ & 0.0 & 28 & 31,34 \\
\hline 5 & 47 & 25 & 36 & 29 & 0 & 0.00 & 0.0 & 26 & 28,27 \\
\hline 6 & 50 & 21 & 36 & 29 & 0 & 0.00 & 0.0 & 13 & 17 \\
\hline 7 & 44 & 25 & 35 & 30 & 0 & 0.73 & 0.0 & 12 & 29 \\
\hline 8 & 48 & 34 & 41 & 24 & 0 & 0.03 & $\mathrm{~T}$ & 25 & 29 \\
\hline 9 & 35 & 23 & 29 & 36 & 0 & 0.00 & 0.0 & 35 & 29 \\
\hline 10 & 48 & 19 & 34 & 31 & 0 & 0.00 & 0.0 & 28 & 20 \\
\hline 11 & 64 & 38 & 51 & 14 & 0 & 0.20 & 0.0 & 39 & 15 \\
\hline 12 & 63 & 31 & 47 & 18 & 0 & 0.75 & $\mathrm{~T}$ & 35 & 26 \\
\hline 13 & 38 & 24 & 31 & 34 & 0 & 0.13 & $1.4^{*}$ & 15 & 20.19 \\
\hline 14 & 45 & 31 & 38 & 27 & 0 & 0.51 & $T$ & 37 & 06 \\
\hline 15 & 47 & 37 & 42 & 23 & 0 & 0.28 & 0.0 & 35 & 21 \\
\hline 16 & 43 & 29 & 36 & 29 & 0 & 0.00 & 0.0 & 21 & 24,25 \\
\hline 17 & 43 & 22 & 33 & 32 & 0 & 0.00 & 0.0 & 22 & 29 \\
\hline 18 & 36 & 22 & 29 & 36 & 0 & 0.13 & 0.4 & 9 & 36,02 \\
\hline 19 & 40 & 34 & 37 & 28 & 0 & 0.21 & 0.0 & 22 & 01 \\
\hline 20 & 41 & 38 & 40 & 25 & 0 & $T$ & 0.0 & 12 & 01 \\
\hline 21 & 53 & 29 & 41 & 24 & 0 & 0.02 & 0.0 & 20 & 27 \\
\hline 22 & 41 & 25 & 33 & 32 & 0 & 0.00 & 0.0 & 27 & 29 \\
\hline 23 & 39 & 28 & 34 & 31 & 0 & 0.00 & 0.0 & 22 & 19 \\
\hline 24 & 39 & 21 & 30 & 35 & 0 & 0.00 & 0.0 & 20 & 36 \\
\hline 25 & 33 & 18 & 26 & 39 & 0 & 0.00 & 0.0 & 11 & 36 \\
\hline 26 & 45 & 20 & 33 & 32 & 0 & 0.00 & 0.0 & 18 & 36 \\
\hline 27 & 45 & 30 & 38 & 27 & 0 & $T$ & 0.0 & 09 & $14 / 07$ \\
\hline 28 & 61 & 32 & 47 & 18 & 0 & 0.00 & 0.0 & 32 & $31 / 25$ \\
\hline 29 & 34 & 20 & 27 & 38 & 0 & 0.45 & $4.9^{*}$ & 20 & 01 \\
\hline 30 & 33 & 9 & 21 & 44 & 0 & 0.00 & 0.0 & 11 & 24 \\
\hline \multicolumn{10}{|l|}{31} \\
\hline Total & $\ldots$ & $\ldots$ & $\ldots$ & 844 & 0 & 4.19 & 6.7 & $\ldots$ & $\ldots$ \\
\hline Mean & 45.0 & 28.4 & 36.9 & - & $\ldots$ & $\ldots$ & $\ldots$ & --- & $\ldots$ \\
\hline Extreme & 64 & 9 & - & $\ldots$ & $\ldots$ & 0.75 & 4.9 & 39 & 15 \\
\hline
\end{tabular}




\begin{tabular}{|c|c|c|c|c|c|c|c|c|c|}
\hline \multicolumn{10}{|c|}{ Daily Climatological Data (Westover AFB, MA-December 1995) } \\
\hline \multirow[b]{3}{*}{ Day } & \multirow[b]{3}{*}{ Max } & \multirow[b]{3}{*}{ Min } & \multicolumn{3}{|c|}{ Temperature (F) } & \multicolumn{2}{|c|}{ Precipitation (in.) } & \multicolumn{2}{|c|}{ Max Wind } \\
\hline & & & \multirow[b]{2}{*}{ Mean $(T)$} & \multicolumn{2}{|c|}{$\begin{array}{l}\text { Degree Days } \\
\left(\text { Base } 65^{\circ} \mathrm{F}\right)\end{array}$} & \multirow{2}{*}{$\begin{array}{l}24 \mathrm{Hr} \\
\text { Water } \\
\text { Equivalent }\end{array}$} & \multirow{2}{*}{$\begin{array}{l}24 \mathrm{Hr} \\
\text { Snowfall } \\
\end{array}$} & \multirow{2}{*}{$\begin{array}{l}\text { Speed } \\
\text { (kts) }\end{array}$} & \multirow[b]{2}{*}{ Direction } \\
\hline & & & & Heating & Cooling & & & & \\
\hline 1 & 43 & 19 & 31 & 34 & $\ldots$ & 0.19 & 1.4 & 31 & 27 \\
\hline 2 & 41 & 23 & 32 & 33 & $\ldots$ & 0.00 & 0.0 & 41 & 27 \\
\hline 3 & 42 & 23 & 33 & 32 & $\ldots$ & 0.03 & 0.0 & 19 & 21 \\
\hline 4 & 47 & 33 & 40 & 25 & $\ldots$ & $T$ & $T$ & 34 & 31 \\
\hline 5 & 37 & 21 & 29 & 36 & $\ldots$ & 0.01 & 0.1 & 18 & 15 \\
\hline 6 & 38 & 28 & 33 & 32 & $\ldots$ & 0.21 & 0.6 & 23 & 29 \\
\hline 7 & 38 & 25 & 32 & 33 & -- & 0.00 & 0.0 & 23 & 31 \\
\hline 8 & 31 & 21 & 26 & 39 & - & 0.00 & 0.0 & 25 & 29 \\
\hline 9 & 31 & 19 & 25 & 40 & - & 0.51 & 3.9 & 24 & 35 \\
\hline 10 & 31 & 4 & 18 & 47 & $\ldots$ & 0.00 & 0.0 & 31 & 28,29 \\
\hline 11 & 23 & 10 & 17 & 48 & - & 0.00 & 0.0 & 28 & 28 \\
\hline 12 & 24 & 5 & 15 & 50 & $\ldots$ & 0.00 & 0.0 & 27 & 29 \\
\hline 13 & 26 & $-2^{*}$ & 12 & 53 & $\ldots$ & 0.00 & 0.0 & 15 & 35 \\
\hline 14 & 20 & 2 & 11 & 54 & - & 0.59 & 6.2 & 01 & 16 \\
\hline 15 & 30 & 21 & 26 & 39 & $\ldots$ & $T$ & $T$ & 12 & 31 \\
\hline 16 & 30 & 27 & 29 & 36 & $\ldots$ & 0.10 & 1.0 & 16 & 32,35 \\
\hline 17 & 36 & 19 & 28 & 37 & $\ldots$ & $T$ & $T$ & 25 & 33 \\
\hline 18 & 34 & 16 & 25 & 40 & $\ldots$ & 0.00 & 0.0 & 18 & 30 \\
\hline 19 & 24 & 15 & 20 & 45 & $\ldots$ & 0.16 & 1.6 & 26 & 36 \\
\hline 20 & 26 & 14 & 20 & 45 & - & 0.26 & 3.6 & 25 & 35,01 \\
\hline 21 & 26 & 13 & 20 & 45 & $\ldots$ & 0.04 & 0.5 & 32 & 31 \\
\hline 22 & 34 & 22 & 33 & 32 & - & 0.00 & 0.0 & 25 & 34 \\
\hline 23 & 34 & 28 & 31 & 34 & $\ldots$ & 0.00 & 0.0 & 21 & 33 \\
\hline 24 & 33 & 16 & 25 & 40 & $\ldots$ & 0.00 & 0.0 & 22 & 30 \\
\hline 25 & 33 & 5 & 19 & 46 & -- & 0.00 & 0.0 & 23 & 33,34 \\
\hline 26 & 32 & 21 & 27 & 38 & - & $\mathrm{T}$ & $T$ & 31 & 31 \\
\hline 27 & 33 & 20 & 27 & 38 & $\ldots$ & $T$ & $T$ & 24 & 35 \\
\hline 28 & 36 & 8 & 22 & 43 & - & 0.00 & 0.0 & 24 & 31 \\
\hline 29 & 33 & 8 & 21 & 44 & -- & 0.00 & 0.0 & 31 & 29 \\
\hline 30 & 38 & 24 & 31 & 34 & $\ldots$ & 0.00 & 0.0 & 20 & 28 \\
\hline 31 & 40 & 24 & 32 & 33 & -- & 0.00 & 0.0 & 12 & $21 / 24 / 20$ \\
\hline Total & $\ldots$ & $\ldots$ & $\ldots$ & 1,225 & $\ldots$ & 2.10 & 18.9 & $\ldots$ & $\ldots$ \\
\hline Mean & 33.0 & 17.2 & 25.5 & $\ldots$ & $\ldots$ & - & - & $\ldots$ & $\ldots$ \\
\hline Extreme & 47 & -2 & $\ldots$ & $\ldots$ & -- & 0.59 & 6.2 & 41 & 27 \\
\hline
\end{tabular}




\begin{tabular}{|c|c|c|c|c|c|c|c|c|c|}
\hline \multicolumn{10}{|c|}{ Daily Climatological Data (Westover AFB, MA-January 1996) } \\
\hline \multirow[b]{3}{*}{ Day } & \multirow[b]{3}{*}{ Max } & \multirow[b]{3}{*}{ Min } & \multicolumn{3}{|c|}{ Temperature (F) } & \multicolumn{2}{|c|}{ Precipitation (in.) } & \multicolumn{2}{|c|}{ Max Wind } \\
\hline & & & \multirow[b]{2}{*}{ Mean $(T)$} & \multicolumn{2}{|c|}{$\begin{array}{l}\text { Degree Days } \\
\left.\text { (Base } 65^{\circ} \mathrm{F}\right)\end{array}$} & \multirow{2}{*}{$\begin{array}{l}24 \mathrm{Hr} \\
\text { Water } \\
\text { Equivalent } \\
\end{array}$} & \multirow[b]{2}{*}{$\begin{array}{l}24 \mathrm{Kr} \\
\text { Snowfall } \\
\end{array}$} & \multirow{2}{*}{$\begin{array}{l}\text { Speed } \\
\text { (kts) }\end{array}$} & \multirow[b]{2}{*}{ Direction } \\
\hline & & & & Heating & Cooling & & & & \\
\hline 1 & 38 & 26 & 32 & 33 & $\ldots$ & $T$ & $T$ & 10 & 35 \\
\hline 2 & 31 & 17 & 24 & 41 & $\ldots$ & 0.24 & 2.7 & 18 & $01 / 36$ \\
\hline 3 & 17 & 15 & 16 & 49 & $\ldots$ & 0.67 & $7.1 *$ & 25 & 35 \\
\hline 4 & 19 & 4 & 12 & 53 & $\ldots$ & 0.02 & 0.2 & 21 & 36 \\
\hline 5 & 18 & -6 & 6 & 59 & $\ldots$ & 0.00 & 0.0 & 42 & 30,29 \\
\hline 6 & 16 & $-10^{*}$ & 3 & 62 & $\ldots$ & 0.00 & 0.0 & 21 & 31 \\
\hline 7 & 12 & 2 & 7 & 58 & $\ldots$ & 0.20 & 2.6 & 27 & 01 \\
\hline 8 & 19 & 3 & 11 & 54 & $\ldots$ & 1.24 & $16.2^{*}$ & 35 & 35,36 \\
\hline 9 & 26 & -3 & 12 & 53 & $\ldots$ & 0.06 & 0.7 & 14 & 18 \\
\hline 10 & 28 & 13 & 21 & 44 & $\ldots$ & 0.05 & 0.8 & 27 & 34 \\
\hline 11 & 27 & 0 & 14 & 51 & $\ldots$ & 0.00 & 0.0 & 18 & 36 \\
\hline 12 & 30 & 9 & 20 & 45 & $\ldots$ & 0.59 & $6.3^{*}$ & 25 & 01,02 \\
\hline 13 & 37 & 26 & 32 & 33 & - & 0.03 & 0.3 & 27 & 33 \\
\hline 14 & 39 & 28 & 34 & 31 & - & 0.00 & 0.0 & 29 & 20 \\
\hline 15 & 39 & 11 & 25 & 40 & $\ldots$ & 0.00 & 0.0 & 31 & 34 \\
\hline 16 & 21 & -1 & 10 & 55 & $\ldots$ & $T$ & 0.0 & 10 & 34,36 \\
\hline 17 & 50 & 22 & 36 & 29 & $\ldots$ & 0.01 & 0.0 & 20 & 18 \\
\hline 18 & 39 & 33 & 36 & 29 & $\ldots$ & 0.00 & 0.0 & 11 & 29,28 \\
\hline 19 & $59 *$ & 25 & 42 & 23 & $\ldots$ & 1.22 & $T$ & 40 & 17 \\
\hline 20 & 30 & 14 & 22 & 43 & $\ldots$ & 0.00 & 0.0 & 25 & 35 \\
\hline 21 & 28 & 10 & 19 & 46 & $\ldots$ & 0.01 & 0.1 & 14 & 02 \\
\hline 22 & 38 & 26 & 32 & 33 & - & $T$ & $T$ & 17 & 17,18 \\
\hline 23 & 39 & 32 & 36 & 29 & $\ldots$ & 0.00 & 0.0 & 24 & 19 \\
\hline 24 & 51 & 36 & 44 & 21 & $\ldots$ & 0.99 & 0.0 & 33 & 20 \\
\hline 25 & 37 & 13 & 25 & 40 & $\ldots$ & 0.00 & 0.0 & 36 & 29 \\
\hline 26 & 34 & 9 & 22 & 43 & $\ldots$ & 0.00 & 0.0 & 15 & 21,23 \\
\hline 27 & 53 & 33 & 43 & 22 & $\ldots$ & 1.59 & $\mathrm{~T}$ & 38 & 15 \\
\hline 28 & 32 & 16 & 24 & 41 & $\ldots$ & $\mathrm{T}$ & $T$ & 38 & 29 \\
\hline 29 & 30 & 11 & 21 & 44 & - & 0.06 & 0.7 & 16 & 17,16 \\
\hline 30 & 43 & 26 & 35 & 30 & $\ldots$ & 0.00 & 0.0 & 21 & 23 \\
\hline 31 & 26 & 9 & 18 & 47 & $\ldots$ & 0.06 & 1.1 & 22 & 35,36 \\
\hline Total & $\ldots$ & $\ldots$ & $\ldots$ & 1,281 & $\ldots$ & 7.04 & 38.8 & $\ldots$ & $\ldots$ \\
\hline Mean & 32.5 & 14.5 & 23.7 & - & $\ldots$ & $\ldots$ & $\ldots$ & $\ldots$ & $\ldots$ \\
\hline Extreme & 59 & -10 & -- & $\ldots$ & $\ldots$ & 1.59 & 16.2 & 42 & 30,29 \\
\hline
\end{tabular}




\begin{tabular}{|c|c|c|c|c|c|c|c|c|c|}
\hline \multirow[b]{3}{*}{ Day } & \multirow[b]{3}{*}{ Max } & \multirow[b]{3}{*}{ Min } & \multicolumn{3}{|c|}{ Temperature (F) } & \multicolumn{2}{|c|}{ Precipitation (in.) } & \multicolumn{2}{|c|}{ Max Wind } \\
\hline & & & \multirow[b]{2}{*}{ Mean (T) } & \multicolumn{2}{|c|}{$\begin{array}{l}\text { Degree Days } \\
\left.\text { (Base } 65^{\circ} \mathrm{F}\right) \\
\end{array}$} & \multirow{2}{*}{$\begin{array}{l}24 \mathrm{Hr} \\
\text { Water } \\
\text { Equivalent } \\
\end{array}$} & \multirow{2}{*}{$\begin{array}{l}24 \mathrm{Hr} \\
\text { Snowfall } \\
\end{array}$} & \multirow{2}{*}{$\begin{array}{l}\text { Speed } \\
\text { (kts) }\end{array}$} & \multirow[b]{2}{*}{ Direction } \\
\hline & & & & Heating & Cooling & & & & \\
\hline 1 & 20 & 0 & 10 & 55 & 0 & 0.00 & 0.0 & 16 & 25 \\
\hline 2 & 28 & 14 & 21 & 44 & 0 & $T$ & 0.3 & 23 & 36 \\
\hline 3 & 18 & 5 & 12 & 53 & 0 & 0.30 & 4.0 & 27 & 36 \\
\hline 4 & 13 & -3 & 5 & 60 & 0 & 0.00 & 0.0 & 22 & 01 \\
\hline 5 & 18 & $-19^{*}$ & -1 & 66 & 0 & 0.00 & 0.0 & 19 & 20 \\
\hline 6 & 22 & $-9 *$ & 7 & 58 & 0 & 0.00 & 0.0 & 23 & 28 \\
\hline 7 & 34 & 1 & 18 & 47 & 0 & 0.00 & 0.0 & 29 & 20 \\
\hline 8 & 36 & 31 & 34 & 31 & 0 & 0.10 & 0.5 & 20 & 17 \\
\hline 9 & 43 & 35 & 39 & 26 & 0 & 0.06 & 0.0 & 30 & 27 \\
\hline 10 & 41 & 31 & 36 & 29 & 0 & 0.00 & 0.0 & 26 & 27 \\
\hline 11 & 45 & 32 & 39 & 26 & 0 & 0.09 & 0.0 & 23 & 33 \\
\hline 12 & 34 & 7 & 21 & 44 & 0 & $T$ & $T$ & 32 & 30 \\
\hline 13 & 15 & 2 & 9 & 56 & 0 & 0.00 & 0.0 & 34 & 31 \\
\hline 14 & 23 & 3 & 13 & 52 & 0 & 0.11 & 2.1 & 16 & 35,01 \\
\hline 15 & 32 & 5 & 19 & 46 & 0 & $T$ & 0.1 & 14 & 28 \\
\hline 16 & 23 & 3 & 13 & 52 & 0 & 0.74 & 8.2 & 30 & 01 \\
\hline 17 & 28 & 20 & 24 & 41 & 0 & 0.05 & 0.8 & 33 & 35 \\
\hline 18 & 28 & 6 & 17 & 48 & 0 & 0.00 & 0.0 & 31 & 28 \\
\hline 19 & 29 & $-1^{*}$ & 14 & 51 & 0 & $T$ & $T$ & 12 & 17 \\
\hline 20 & 49 & 27 & 38 & 27 & 0 & $T$ & 0.0 & 15 & 15,16 \\
\hline 21 & 48 & 41 & 45 & 20 & 0 & 0.55 & 0.0 & $M$ & $\mathrm{M}$ \\
\hline 22 & 51 & 38 & 45 & 20 & 0 & 0.67 & 0.0 & $M$ & $M$ \\
\hline 23 & 51 & 33 & 42 & 23 & 0 & 0.00 & 0.0 & 13 & 36 \\
\hline 24 & 46 & 36 & 41 & 24 & 0 & 0.18 & 0.0 & 35 & 26 \\
\hline 25 & 52 & 41 & 47 & 18 & 0 & 0.00 & 0.0 & 53 & 33 \\
\hline 26 & 52 & 29 & 41 & 24 & 0 & 0.00 & 0.0 & 31 & 32 \\
\hline 27 & 54 & 23 & 39 & 26 & 0 & 0.02 & 0.0 & 20 & 35 \\
\hline 28 & 49 & 30 & 40 & 25 & 0 & 0.13 & $T$ & 36 & 29 \\
\hline 29 & 29 & 18 & 24 & 41 & 0 & 0.00 & 0.0 & 36 & 28 \\
\hline \multicolumn{10}{|l|}{30} \\
\hline \multicolumn{10}{|l|}{31} \\
\hline Total & $\ldots$ & $\ldots$ & $\ldots$ & 1,133 & 0 & 3.00 & 16.0 & $\ldots$ & -- \\
\hline Mean & 34.9 & 16.5 & 25.9 & $\ldots$ & $\ldots$ & $\ldots$ & $\ldots$ & $\ldots$ & -- \\
\hline Extreme & 54 & -19 & $\ldots$ & $\ldots$ & $\ldots$ & 0.74 & 8.2 & 53 & 33 \\
\hline
\end{tabular}




\begin{tabular}{|c|c|c|c|c|c|c|c|c|c|}
\hline \multicolumn{10}{|c|}{ Daily Climatological Data (Westover AFB, MA-March 1996) } \\
\hline \multirow[b]{3}{*}{ Day } & \multirow[b]{3}{*}{ Max } & \multirow[b]{3}{*}{ Min } & \multicolumn{3}{|c|}{ Temperature (F) } & \multicolumn{2}{|c|}{ Precipitation (in.) } & \multicolumn{2}{|c|}{ Max Wind } \\
\hline & & & \multirow[b]{2}{*}{ Mean $(T)$} & \multicolumn{2}{|c|}{$\begin{array}{l}\text { Degree Days } \\
\left(\text { Base } 65^{\circ} \mathrm{F}\right)\end{array}$} & \multirow{2}{*}{$\begin{array}{l}24 \mathrm{Hr} \\
\text { Water } \\
\text { Equivalent } \\
\end{array}$} & \multirow{2}{*}{\begin{tabular}{|l}
$24 \mathrm{Hr}$ \\
Snowfall \\
\end{tabular}} & \multirow{2}{*}{$\begin{array}{l}\text { Speed } \\
\text { (kts) } \\
\end{array}$} & \multirow[b]{2}{*}{ Direction } \\
\hline & & & & Heating & Cooling & & & & \\
\hline 1 & 34 & 12 & 23 & 42 & 0 & 0.00 & 0.0 & 29 & 21 \\
\hline 2 & 28 & 23 & 26 & 39 & 0 & 0.56 & 5.3 & 15 & 35,36 \\
\hline 3 & 36 & 20 & 28 & 37 & 0 & 0.07 & 0.9 & 38 & 28 \\
\hline 4 & 30 & 11 & 21 & 44 & 0 & 0.03 & 0.3 & 35 & 29 \\
\hline 5 & 33 & 10 & 22 & 43 & 0 & 0.24 & 1.2 & 9 & 35 \\
\hline 6 & 33 & 29 & 31 & 34 & 0 & 0.14 & 1.0 & 18 & $35,01,36$ \\
\hline 7 & 29 & 22 & 26 & 39 & 0 & 0.41 & 6.0 & 21 & 01,36 \\
\hline 8 & 25 & 12 & 19 & 46 & 0 & 0.35 & 4.5 & 27 & 36 \\
\hline 9 & 21 & 4 & 13 & 52 & 0 & 0.00 & 0.0 & 34 & 28 \\
\hline 10 & 35 & $-8^{*}$ & 14 & 51 & 0 & 0.00 & 0.0 & $M$ & $M$ \\
\hline 11 & 43 & $1 *$ & 22 & 43 & 0 & 0.00 & 0.0 & 12 & 28,27 \\
\hline 12 & 49 & $7 *$ & 28 & 37 & 0 & 0.00 & 0.0 & 18 & 33 \\
\hline 13 & 56 & 16 & 36 & 29 & 0 & 0.00 & 0.0 & 10 & 25 \\
\hline 14 & 61 & 27 & 44 & 21 & 0 & 0.00 & 0.0 & 17 & 20 \\
\hline 15 & 49 & 35 & 42 & 23 & 0 & 0.32 & 0.0 & 17 & 30 \\
\hline 16 & 39 & 27 & 33 & 32 & 0 & 0.00 & 0.0 & 32 & 33 \\
\hline 17 & 53 & 17 & 35 & 30 & 0 & 0.00 & 0.0 & 17 & 27 \\
\hline 18 & 55 & 23 & 39 & 26 & 0 & 0.00 & 0.0 & 13 & 18 \\
\hline 19 & 57 & 26 & 42 & 23 & 0 & $\mathrm{~T}$ & 0.0 & 37 & 07 \\
\hline 20 & 46 & 35 & 41 & 24 & 0 & 0.33 & 0.0 & 30 & 06 \\
\hline 21 & 46 & 35 & 41 & 24 & 0 & 0.05 & 0.0 & 18 & 19 \\
\hline 22 & 41 & 30 & 36 & 29 & 0 & 0.00 & 0.0 & 24 & 28 \\
\hline 23 & 37 & 25 & 31 & 34 & 0 & $T$ & $T$ & 37 & 29 \\
\hline 24 & 50 & 27 & 39 & 26 & 0 & 0.00 & 0.0 & 25 & 29,34 \\
\hline 25 & 68 & 27 & 48 & 17 & 0 & 0.00 & 0.0 & 32 & 20 \\
\hline 26 & 57 & 31 & 44 & 21 & 0 & $T$ & 0.0 & 26 & $23,25,29$ \\
\hline 27 & 37 & 24 & 31 & 34 & 0 & $I$ & I & 33 & 28,29 \\
\hline 28 & 43 & 16 & 30 & 35 & 0 & 0.00 & 0.0 & 16 & 27 \\
\hline 29 & 49 & 28 & 39 & 26 & 0 & 0.00 & 0.0 & 19 & 05,04 \\
\hline 30 & 53 & 23 & 38 & 27 & 0 & 0.00 & 0.0 & 28 & 36 \\
\hline 31 & 62 & 21 & 42 & 23 & 0 & 0.00 & 0.0 & 16 & 35 \\
\hline Total & $\ldots$ & $\ldots$ & $\ldots$ & 1,011 & 0 & 2.50 & 19.2 & $\ldots$ & $\ldots$ \\
\hline Mean & 43.7 & 20.5 & 32.4 & - & - & $\ldots$ & $\ldots$ & $\ldots$ & - \\
\hline Extreme & 68 & -8 & $\ldots$ & $\ldots$ & $\ldots$ & 0.56 & 6.0 & 38 & 28 \\
\hline
\end{tabular}




\begin{tabular}{|c|c|c|c|c|c|c|c|c|c|}
\hline \multicolumn{10}{|c|}{ Daily Climatological Data (Westover AFB, MA-April 1996) } \\
\hline \multirow[b]{3}{*}{ Day } & \multirow[b]{3}{*}{ Max } & \multicolumn{4}{|c|}{ Temperature (F) } & \multicolumn{2}{|c|}{ Precipitation (in.) } & \multicolumn{2}{|c|}{ Max Wind } \\
\hline & & \multirow[b]{2}{*}{ Min } & \multirow[b]{2}{*}{ Mean $(T)$} & \multicolumn{2}{|c|}{$\begin{array}{l}\text { Degree Days } \\
\left.\text { (Base } 65^{\circ} \mathrm{F}\right)\end{array}$} & \multirow{2}{*}{$\begin{array}{l}24 \mathrm{Hr} \\
\text { Water } \\
\text { Equivalent } \\
\end{array}$} & \multirow{2}{*}{$\begin{array}{l}24 \mathrm{Hr} \\
\text { Snowfall } \\
\end{array}$} & \multirow{2}{*}{$\begin{array}{l}\text { Speed } \\
\text { (kts) } \\
\end{array}$} & \multirow[b]{2}{*}{ Direction } \\
\hline & & & & Heating & Cooling & & & & \\
\hline 1 & 59 & 29 & 44 & 21 & $\ldots$ & 0.20 & 0.0 & 19 & 20 \\
\hline 2 & 50 & 37 & 44 & 21 & $\ldots$ & 0.59 & 0.0 & 41 & 36 \\
\hline 3 & 51 & 30 & 41 & 24 & $\ldots$ & 0.00 & 0.0 & 32 & 29 \\
\hline 4 & 50 & 29 & 40 & 25 & $\ldots$ & 0.00 & 0.0 & 23 & 26 \\
\hline 5 & 53 & 38 & 46 & 19 & $\ldots$ & $T$ & $T$ & 22 & 02 \\
\hline 6 & 52 & 31 & 42 & 23 & $\ldots$ & $\mathrm{T}$ & 0.0 & 22 & 06 \\
\hline 7 & 43 & 33 & 38 & 27 & $\ldots$ & 0.14 & 0.6 & 29 & 07 \\
\hline 8 & 43 & 30 & 37 & 28 & $\ldots$ & 0.09 & 0.6 & 21 & 34 \\
\hline 9 & 46 & 25 & 36 & 29 & $\ldots$ & 0.19 & 1.2 & 19 & 06 \\
\hline 10 & 40 & 31 & 36 & 29 & $\ldots$ & 0.69 & $5.7^{*}$ & 38 & 33 \\
\hline 11 & 59 & 39 & 49 & 16 & $\ldots$ & 0.00 & 0.0 & 37 & 35 \\
\hline 12 & 61 & 46 & 54 & 11 & $\ldots$ & 0.02 & 0.0 & 27 & $30 / 28$ \\
\hline 13 & 49 & 37 & 43 & 22 & $\ldots$ & 0.36 & 0.0 & 20 & 36 \\
\hline 14 & 42 & 35 & 39 & 26 & $\ldots$ & 0.29 & 0.0 & .24 & 34,33 \\
\hline 15 & 60 & 29 & 45 & 20 & $\ldots$ & 0.00 & 0.0 & 20 & 15,17 \\
\hline 16 & 47 & 38 & 43 & 22 & $\ldots$ & $3.09^{*}$ & $T$ & 26 & 28,27 \\
\hline 17 & 48 & 39 & 44 & 21 & $\ldots$ & $T$ & $T$ & 31 & 27 \\
\hline 18 & 62 & 35 & 49 & 16 & $\ldots$ & 0.00 & 0.0 & 28 & 33 \\
\hline 19 & 71 & 32 & 52 & 13 & $\ldots$ & 0.00 & 0.0 & 25 & 20 \\
\hline 20 & 74 & 42 & 58 & 7 & $\ldots$ & 0.00 & 0.0 & 26 & $19 / 20$ \\
\hline 21 & 79 & 46 & 63 & 2 &.- & 0.00 & 0.0 & 23 & $33 / 29$ \\
\hline 22 & 78 & 40 & 59 & 6 & $\ldots$ & 0.02 & 0.0 & 27 & 21 \\
\hline 23 & $83^{*}$ & 53 & 68 & $\ldots$ & 3 & 0.57 & 0.0 & 29 & $22 / 21$ \\
\hline 24 & 61 & 34 & 48 & 17 & $\ldots$ & $T$ & 0.0 & 46 & 29 \\
\hline 25 & 70 & 35 & 53 & 12 & $\ldots$ & 0.00 & 0.0 & 29 & $22 / 23$ \\
\hline 26 & 70 & 55 & 63 & 2 & - & 0.23 & 0.0 & 29 & $17 / 19$ \\
\hline 27 & 55 & 30 & 43 & 22 & $\ldots$ & 0.00 & 0.0 & 29 & 34 \\
\hline 28 & 63 & 27 & 45 & 20 & $\ldots$ & 0.00 & 0.0 & 30 & 29 \\
\hline 29 & 46 & 39 & 43 & 22 & $\ldots$ & $1.27^{*}$ & 0.0 & 19 & 36 \\
\hline 30 & 68 & 43 & 56 & 9 & $\ldots$ & 0.61 & 0.0 & 32 & 21 \\
\hline 31 & $\ldots$ & - & $\ldots$ & $\ldots$ & $\ldots$ & - & $\ldots$ & - & $\ldots$ \\
\hline Total & $\ldots$ & $\ldots$ & $\ldots$ & 532 & 3 & 8.34 & 8.1 & $\ldots$ & $\ldots$ \\
\hline Mean & 57.8 & 36.2 & 47.4 & - & - & - & $\ldots$ & - & $\ldots$ \\
\hline Extreme & 83 & 25 & $\ldots$ & $\ldots$ & - & $3.09^{*}$ & $5.7^{*}$ & 41 & 36 \\
\hline
\end{tabular}




\begin{tabular}{|c|c|c|c|c|c|c|c|c|c|}
\hline \multicolumn{10}{|c|}{ Daily Climatological Data (Westover AFB, MA-May 1996) } \\
\hline \multirow[b]{3}{*}{ Day } & \multirow[b]{3}{*}{ Max } & \multirow[b]{3}{*}{ Min } & \multicolumn{3}{|c|}{ Temperature (F) } & \multicolumn{2}{|c|}{ Precipitation (in.) } & \multicolumn{2}{|c|}{ Max Wind } \\
\hline & & & \multirow[b]{2}{*}{ Mean $(T)$} & \multicolumn{2}{|c|}{$\begin{array}{l}\text { Degree Days } \\
\left(\text { Base } 65^{\circ} \mathrm{F}\right)\end{array}$} & \multirow{2}{*}{$\begin{array}{l}24 \mathrm{Hr} \\
\text { Water } \\
\text { Equivalent } \\
\end{array}$} & \multirow{2}{*}{$\begin{array}{l}24 \mathrm{Hr} \\
\text { Snowfall } \\
\end{array}$} & \multirow{2}{*}{$\begin{array}{l}\text { Speed } \\
\text { (kts) }\end{array}$} & \multirow[b]{2}{*}{ Direction } \\
\hline & & & & Heating & Cooling & & & & \\
\hline 1 & 68 & 47 & 58 & 7 & -- & 0.00 & 0 & 25 & 20 \\
\hline 2 & 66 & 38 & 52 & 13 & -- & $T$ & 0 & 31 & 26 \\
\hline 3 & 54 & 34 & 44 & 21 & -- & 0.32 & 0 & 16 & 19 \\
\hline 4 & 55 & 50 & 53 & 12 & - & 0.15 & 0 & 17 & 01,35 \\
\hline 5 & 68 & 42 & 55 & 10 & $\ldots$ & 0.05 & 0 & 22 & 30,36 \\
\hline 6 & 50 & 30 & 40 & 25 & $\ldots$ & 0.31 & 0 & 24 & 05 \\
\hline 7 & 66 & $27 * T$ & 47 & 18 & $\ldots$ & 0.00 & 0 & 26 & 18 \\
\hline 8 & 68 & 44 & 56 & 9 & -- & 0.04 & 0 & 16 & 18 \\
\hline 9 & 66 & 48 & 57 & 8 & $\ldots$ & $\mathrm{T}$ & 0 & 21 & 19,17 \\
\hline 10 & 63 & 49 & 56 & 9 & - & 0.30 & 0 & 16 & 18 \\
\hline 11 & 81 & 48 & 65 & $\ldots$ & - & $1.31^{*}$ & 0 & 42 & 34 \\
\hline 12 & 49 & 42 & 46 & 19 & $\ldots$ & 0.13 & 0 & 34 & 27,31 \\
\hline 13 & 53 & 29 & 41 & 24 & - & 0.00 & 0 & 28 & 27 \\
\hline 14 & 61 & $27^{*}$ & 44 & 21 & $\ldots$ & 0.00 & 0 & 25 & 30 \\
\hline 15 & 71 & $30 * T$ & 51 & 14 & $\ldots$ & 0.00 & 0 & 20 & 19,21 \\
\hline 16 & 53 & $34^{*}$ & 44 & 21 & $\ldots$ & 0.56 & 0 & 17 & 16 \\
\hline 17 & 70 & 45 & 58 & 7 & $\ldots$ & 0.02 & 0 & 21 & 34 \\
\hline 18 & 64 & 46 & 55 & 10 & $\ldots$ & 0.03 & 0 & 18 & 20 \\
\hline 19 & 84 & 53 & 69 & $\ldots$ & 4 & 0.00 & 0 & 20 & 22 \\
\hline 20 & $92^{*}$ & 60 & 76 & $\ldots$ & 11 & 0.00 & 0 & 30 & 27 \\
\hline 21 & 87 & 60 & 74 & $\ldots$ & 9 & 0.09 & 0 & 30 & 29 \\
\hline 22 & 77 & 48 & 63 & 2 & $\ldots$ & $\ldots$ & 0 & 32 & 27 \\
\hline 23 & 78 & 45 & 62 & 3 & $\ldots$ & $\ldots$ & 0 & 27 & 26 \\
\hline 24 & 70 & 50 & 60 & 5 & - & $T$ & 0 & 28 & 33 \\
\hline 25 & 66 & 37 & 52 & 13 & $\ldots$ & 0.00 & 0 & 25 & 01,35 \\
\hline 26 & 69 & 36 & 53 & 12 & $\ldots$ & 0.00 & 0 & 30 & 29 \\
\hline 27 & 67 & 49 & 58 & 7 & $\ldots$ & 0.00 & 0 & 15 & 25 \\
\hline 28 & 67 & 49 & 58 & 7 & $\ldots$ & 0.00 & 0 & 18 & 18,21 \\
\hline 29 & 67 & 45 & 56 & 9 & $\ldots$ & 0.00 & 0 & 17 & 04,35 \\
\hline 30 & 57 & 44 & 51 & 14 & - & 0.03 & 0 & 30 & 36 \\
\hline 31 & 75 & 34 & 55 & 10 & $\ldots$ & 0.00 & 0 & 25 & 27 \\
\hline Total & $\ldots$ & $\ldots$ & $\ldots$ & 330 & 24 & 3.34 & 0 & $\ldots$ & $\ldots$ \\
\hline Mean & 67.2 & 42.6 & 55.1 & $\ldots$ & $\ldots$ & -- & $\ldots$ & $\ldots$ & $\ldots$ \\
\hline Extreme & 92 & 27 & $\ldots$ & $\cdots$ & $\ldots$ & 1.31 & 0 & 42 & 34 \\
\hline
\end{tabular}




\begin{tabular}{|c|c|c|c|c|c|c|c|c|c|}
\hline \multicolumn{10}{|c|}{ Daily Climatological Data (Westover AFB, MA-June 1996) } \\
\hline \multirow[b]{3}{*}{ Day } & \multirow[b]{3}{*}{ Max } & \multirow[b]{3}{*}{ Min } & \multicolumn{3}{|c|}{ Temperature (F) } & \multicolumn{2}{|c|}{ Precipitation (in.) } & \multicolumn{2}{|c|}{ Max Wind } \\
\hline & & & \multirow[b]{2}{*}{ Mean (T) } & \multicolumn{2}{|c|}{$\begin{array}{l}\text { Degree Days } \\
\left.\text { (Base } 65^{\circ} \mathrm{F}\right)\end{array}$} & \multirow{2}{*}{$\begin{array}{l}24 \mathrm{Hr} \\
\text { Water } \\
\text { Equivalent } \\
\end{array}$} & \multirow{2}{*}{\begin{tabular}{|l}
$24 \mathrm{Hr}$ \\
Snowfall \\
\end{tabular}} & \multirow{2}{*}{$\begin{array}{l}\text { Speed } \\
\text { (kts) }\end{array}$} & \multirow[b]{2}{*}{ Direction } \\
\hline & & & & Heating & Cooling & & & & \\
\hline 1 & 79 & 39 & 59 & 6 & $\ldots$ & 0.00 & 0 & 17 & 35 \\
\hline 2 & 77 & 43 & 60 & 5 & $\ldots$ & 0.00 & 0 & 21 & 23,18 \\
\hline 3 & 62 & 40 & 51 & 14 & $\ldots$ & 0.75 & 0 & 15 & 05 \\
\hline 4 & 75 & 59 & 67 & $\ldots$ & 2 & 0.16 & 0 & $M$ & $M$ \\
\hline 5 & 79 & 52 & 66 & $\ldots$ & 1 & $T$ & 0 & $M$ & $M$ \\
\hline 6 & 79 & 48 & 64 & 1 & $\ldots$ & 0.00 & 0 & $M$ & $M$ \\
\hline 7 & 80 & 55 & 68 & $\ldots$ & 3 & 0.00 & 0 & $M$ & $M$ \\
\hline 8 & 83 & 64 & 74 & $\ldots$ & 9 & 0.28 & 0 & $M$ & $M$ \\
\hline 9 & 79 & 63 & 71 & $\ldots$ & 6 & 0.01 & 0 & $M$ & $M$ \\
\hline 10 & 76 & 62 & 69 & - & 4 & 0.27 & 0 & $M$ & $M$ \\
\hline 11 & 82 & 68 & 75 & $\ldots$ & 10 & 0.00 & 0 & $M$ & $M$ \\
\hline 12 & 81 & 68 & 75 & $\ldots$ & 10 & 0.00 & 0 & $M$ & $M$ \\
\hline 13 & 80 & 61 & 71 & $\ldots$ & 6 & 0.31 & 0 & 23 & 28 \\
\hline 14 & 84 & 58 & 70 & $\ldots$ & 5 & 0.00 & 0 & 19 & 28 \\
\hline 15 & 86 & 58 & 72 & $\ldots$ & 7 & 0.00 & 0 & 17 & 29 \\
\hline 16 & 80 & 57 & 69 & - & 4 & 0.00 & 0 & 18 & 22 \\
\hline 17 & 81 & 59 & 70 & $\ldots$ & 5 & 0.01 & 0 & 11 & 19,21 \\
\hline 18 & 75 & 57 & 66 & $\ldots$ & 1 & $T$ & 0 & 16 & 05 \\
\hline 19 & 69 & 52 & 61 & 4 & - & 0.23 & 0 & 11 & 21 \\
\hline 20 & 67 & 57 & 62 & 3 & - & 0.38 & 0 & 07 & 04 \\
\hline 21 & 81 & 61 & 71 & - & 6 & 0.05 & 0 & 19 & 30,28 \\
\hline 22 & 73 & 57 & 65 & - & -- & 0.03 & 0 & 16 & 16 \\
\hline 23 & 80 & 56 & 68 & - & 3 & 0.00 & 0 & 25 & $33,34,36$ \\
\hline 24 & 74 & 54 & 64 & 1 & $\ldots$ & $T$ & 0 & 16 & 18 \\
\hline 25 & 82 & 62 & 72 & - & 7 & 0.02 & 0 & 23 & 31,30 \\
\hline 26 & 76 & 53 & 65 & $\ldots$ & $\ldots$ & 0.00 & 0 & 23 & 35 \\
\hline 27 & 79 & 45 & 62 & 3 & - & $T$ & 0 & 16 & 36,31 \\
\hline 28 & 78 & 50 & 64 & 1 & $\ldots$ & 0.00 & 0 & 20 & 02 \\
\hline 29 & 73 & 50 & 62 & 3 & $\ldots$ & $T$ & 0 & 15 & 19 \\
\hline 30 & 64 & 61 & 63 & 2 & - & 0.06 & 0 & 20 & 18 \\
\hline \multicolumn{10}{|l|}{31} \\
\hline Total & $\ldots$ & $\ldots$ & - & 42 & 89 & 2.56 & 0 & $\ldots$ & -- \\
\hline Mean & 77.1 & 55.6 & 66.5 & $\ldots$ & $\ldots$ & $\ldots$ & $-\ldots$ & - & $-\ldots$ \\
\hline Extreme & 86 & 39 & $\ldots$ & $\ldots$ & $\ldots$ & 0.75 & 0 & 25 & 34 \\
\hline
\end{tabular}




\begin{tabular}{|c|c|c|c|c|c|c|c|c|c|}
\hline \multicolumn{10}{|c|}{ Daily Climatological Data (Westover AFB, MA-July 1996) } \\
\hline \multirow[b]{3}{*}{ Day } & \multirow[b]{3}{*}{ Max } & \multirow[b]{3}{*}{ Min } & \multicolumn{3}{|c|}{ Temperature (F) } & \multicolumn{2}{|c|}{ Precipitation (in.) } & \multicolumn{2}{|c|}{ Max Wind } \\
\hline & & & \multirow[b]{2}{*}{ Mean $(T)$} & \multicolumn{2}{|c|}{$\begin{array}{l}\text { Degree Days } \\
\left.\text { (Base } 65^{\circ} \mathrm{F}\right)\end{array}$} & \multirow{2}{*}{$\begin{array}{l}24 \mathrm{Hr} \\
\text { Water } \\
\text { Equivalent } \\
\end{array}$} & \multirow[b]{2}{*}{$\begin{array}{l}24 \mathrm{Hr} \\
\text { Snowfall } \\
\end{array}$} & \multirow[b]{2}{*}{$\begin{array}{l}\text { Speed } \\
\text { (kts) }\end{array}$} & \multirow[b]{2}{*}{ Direction } \\
\hline & & & & Heating & Cooling & & & & \\
\hline 1 & 88 & 63 & 76 & $\ldots$ & 11 & 0.00 & 0 & 17 & $25 / 19$ \\
\hline 2 & 86 & 57 & 72 & $\ldots$ & 7 & 0.00 & 0 & 14 & $25 / 27$ \\
\hline 3 & 72 & 61 & 67 & $\ldots$ & 2 & 1.12 & 0 & 22 & 22 \\
\hline 4 & 75 & 61 & 68 & $\ldots$ & 3 & 0.12 & 0 & 20 & 25 \\
\hline 5 & 82 & 61 & 72 & $\ldots$ & 7 & 0.00 & 0 & 23 & 28 \\
\hline 6 & 86 & 54 & 70 & $\ldots$ & 5 & 0.00 & 0 & 20 & 24 \\
\hline 7 & 81 & 57 & 69 & $\ldots$ & 4 & 0.00 & 0 & 17 & 21 \\
\hline 8 & 84 & 64 & 74 & $\ldots$ & 9 & 0.35 & 0 & 20 & 18,21 \\
\hline 9 & 84 & 64 & 74 & $\ldots$ & 9 & 0.40 & 0 & 21 & 29 \\
\hline 10 & 75 & 59 & 67 & -- & 2 & 0.00 & 0 & 20 & 34,32 \\
\hline 11 & 79 & 50 & 65 & $\ldots$ & - & 0.00 & 0 & 19 & 25 \\
\hline 12 & 79 & 52 & 64 & 1 & $\ldots$ & $T$ & 0 & 18 & 19,18 \\
\hline 13 & 70 & 61 & 66 & -- & 1 & $3.09^{*}$ & 0 & 24 & 04 \\
\hline 14 & 86 & 64 & 75 & $\ldots$ & 10 & 0.00 & 0 & 16 & 27 \\
\hline 15 & 75 & 70 & 73 & $\ldots$ & 8 & 0.60 & 0 & 24 & 20 \\
\hline 16 & 84 & 64 & 74 & -- & 9 & 0.19 & 0. & 22 & 24 \\
\hline 17 & 86 & 64 & 75 & $\ldots$ & 10 & 0.00 & 0 & 19 & 25 \\
\hline 18 & 86 & 57 & 72 & -- & 7 & 0.00 & 0 & 17 & 26 \\
\hline 19 & 81 & 66 & 74 &.-- & 9 & 0.27 & 0 & 22 & 33 \\
\hline 20 & 73 & 61 & 67 & -- & 2 & 0.00 & 0 & 30 & 32 \\
\hline 21 & 82 & 57 & 70 & - & 5 & 0.00 & 0 & 22 & $29 / 32$ \\
\hline 22 & 81 & 52 & 67 & $\ldots$ & 2 & 0.00 & 0 & 15 & 21 \\
\hline 23 & 64 & 59 & 62 & 3 & -- & 0.40 & 0 & 11 & 20 \\
\hline 24 & 75 & 63 & 69 & - & 4 & 0.00 & 0 & 12 & 19 \\
\hline 25 & 72 & 64 & 68 & $\ldots$ & 3 & 0.03 & 0 & 14 & 18 \\
\hline 26 & 77 & 63 & 70 & --. & 5 & 0.19 & 0 & 10 & $\begin{array}{l}01,02,03, \\
16,19\end{array}$ \\
\hline 27 & 79 & 59 & 69 & $\ldots$ & 4 & 0.00 & 0 & 19 & 29 \\
\hline 28 & 82 & 54 & 68 & $\ldots$ & 3 & 0.00 & 0 & 12 & 32 \\
\hline 29 & 79 & 55 & 67 & $\ldots$ & 2 & 0.00 & 0 & 11 & 24,23 \\
\hline 30 & 73 & 57 & 65 & $\ldots$ & $\ldots$ & 0.00 & 0 & 11 & 10 \\
\hline 31 & 64 & 59 & 62 & 3 & $\ldots$ & 0.13 & 0 & 10 & 15 \\
\hline Total & -- & $\ldots$ & $\ldots$ & 7 & 143 & 6.89 & 0 & $\ldots$ & $\ldots$ \\
\hline Mean & 78.7 & 59.7 & 69.4 & $\ldots$ & - & $\ldots$ & $\ldots$ & - & $\ldots$ \\
\hline Extreme & 88 & 50 & $\ldots$ & $\ldots$ & $\ldots$ & 3.09 & 0 & 30 & 32 \\
\hline
\end{tabular}




\begin{tabular}{|c|c|c|c|c|c|c|c|c|c|}
\hline \multicolumn{10}{|c|}{ Daily Climatological Data (Westover AFB, MA-August 1996) } \\
\hline \multirow[b]{3}{*}{ Day } & \multirow[b]{3}{*}{ Max } & \multirow[b]{3}{*}{ Min } & \multicolumn{3}{|c|}{ Temperature (F) } & \multicolumn{2}{|c|}{ Precipitation (in.) } & \multicolumn{2}{|c|}{ Max Wind } \\
\hline & & & \multirow[b]{2}{*}{ Mean $(T)$} & \multicolumn{2}{|c|}{$\begin{array}{l}\text { Degree Days } \\
\left(\text { Base } 65^{\circ} \mathrm{F}\right)\end{array}$} & \multirow{2}{*}{$\begin{array}{l}24 \mathrm{Hr} \\
\text { Water } \\
\text { Equivalent } \\
\end{array}$} & \multirow{2}{*}{$\begin{array}{l}24 \mathrm{Hr} \\
\text { Snowfall } \\
\end{array}$} & \multirow{2}{*}{$\begin{array}{l}\text { Speed } \\
\text { (kts) }\end{array}$} & \multirow[b]{2}{*}{ Direction } \\
\hline & & & & Heating & Cooling & & & & \\
\hline 1 & 68 & 61 & 65 & $\ldots$ & $\ldots$ & $\mathrm{T}$ & 0 & 7 & 12,03 \\
\hline 2 & 81 & 61 & 71 & -- & 6 & 0.02 & 0 & 16 & 22 \\
\hline 3 & 84 & 64 & 74 & $\ldots$ & 9 & 0.00 & 0 & 13 & 23 \\
\hline 4 & 86 & 64 & 75 & $\ldots$ & 10 & 0.00 & 0 & 13 & 33 \\
\hline 5 & 86 & 64 & 75 & $\ldots$ & 10 & 0.00 & 0 & 18 & 02 \\
\hline 6 & 88 & 61 & 75 & $\ldots$ & 10 & 0.00 & 0 & 10 & 07 \\
\hline 7 & 86 & 70 & 78 & $\ldots$ & 13 & 0.00 & 0 & 13 & 20 \\
\hline 8 & 84 & 66 & 75 & $\ldots$ & 10 & 0.00 & 0 & 18 & 22 \\
\hline 9 & 84 & 67 & 76 & $\ldots$ & 11 & $\mathrm{~T}$ & 0 & 16 & 31,32 \\
\hline 10 & 82 & 61 & 72 & $\ldots$ & 7 & 0.00 & 0 & 18 & 35 \\
\hline 11 & 77 & 57 & 67 & $\ldots$ & 2 & 0.00 & 0 & 16 & 35,36 \\
\hline 12 & 73 & 57 & 65 & $\ldots$ & $\ldots$ & $T$ & 0 & 13 & 18 \\
\hline 13 & 70 & 54 & 62 & 3 & $\ldots$ & 0.07 & 0 & 12 & 01,33 \\
\hline 14 & 84 & 52 & 63 & & 3 & 0.00 & 0 & 10 & 01,05 \\
\hline 15 & 82 & 59 & 71 & $\ldots$ & 6 & 0.00 & 0 & 16 & 20 \\
\hline 16 & 75 & 55 & 65 & $\ldots$ & $\ldots$ & 0.00 & 0 & 14 & $18,19,21$ \\
\hline 17 & 84 & 59 & 72 & $\ldots$ & 7 & 0.00 & 0 & 24 & 33 \\
\hline 18 & 86 & 59 & 73 & $\ldots$ & 8 & 0.00 & 0 & 17 & 36 \\
\hline 19 & 84 & 55 & 70 & - & 5 & 0.00 & 0 & 16 & 36 \\
\hline 20 & 81 & 64 & 73 & -- & 8 & 0.00 & 0 & 16 & 18,20 \\
\hline 21 & 82 & 64 & 73 & $\ldots$ & 8 & 0.04 & 0 & 14 & 25,28 \\
\hline 22 & 88 & 59 & 74 & - & 9 & 0.00 & 0 & 10 & 23,25 \\
\hline 23 & $90^{*}$ & 61 & 76 & $\ldots$ & 11 & 0.14 & 0 & 29 & 28 \\
\hline 24 & 81 & 63 & 72 & - & 7 & 0.40 & 0 & 15 & 36 \\
\hline 25 & 82 & 63 & 73 & $\ldots$ & 8 & 0.00 & 0 & 17 & 22 \\
\hline 26 & 86 & 59 & 73 & - & 8 & 0.00 & 0 & 16 & 19 \\
\hline 27 & 81 & 59 & 70 & -- & 5 & 0.81 & 0 & 18 & 27 \\
\hline 28 & 72 & 59 & 66 & -- & 1 & 0.00 & 0 & 11 & 02,36 \\
\hline 29 & 81 & 55 & 68 & $\ldots$ & 3 & 0.00 & 0 & 20 & 30 \\
\hline 30 & 79 & 50 & 65 & -- & $\ldots$ & 0.00 & 0 & 14 & 06 \\
\hline 31 & 81 & 46 & 64 & 1 & - & 0.00 & 0 & 10 & 23 \\
\hline Total & $\ldots$ & $\ldots$ & $\ldots$ & 4 & 85 & 1.48 & 0 & - & - \\
\hline Mean & 81.5 & 59.6 & 70.6 & - & - & - & $\ldots$ & - & $\ldots$ \\
\hline Extreme & 90 & 46 & $\ldots$ & $\ldots$ & $\ldots$ & 0.81 & 0 & 29 & 28 \\
\hline
\end{tabular}




\begin{tabular}{|c|c|c|c|c|c|c|c|c|c|}
\hline \multicolumn{10}{|c|}{ Daily Climatological Data (Westover AFB, MA-September 1996) } \\
\hline \multirow[b]{3}{*}{ Day } & \multirow[b]{3}{*}{ Max } & \multirow[b]{3}{*}{ Min } & \multicolumn{3}{|c|}{ Temperature (F) } & \multicolumn{2}{|c|}{ Precipitation (in.) } & \multicolumn{2}{|c|}{ Max Wind } \\
\hline & & & \multirow[b]{2}{*}{ Mean ( $($ ) } & \multicolumn{2}{|c|}{$\begin{array}{l}\text { Degree Days } \\
\left(\text { Base } 65^{\circ} \mathrm{F}\right)\end{array}$} & \multirow{2}{*}{\begin{tabular}{|l}
$24 \mathrm{Hr}$ \\
Water \\
Equivalent \\
\end{tabular}} & \multirow[b]{2}{*}{$\begin{array}{l}24 \mathrm{Hr} \\
\text { Snowfall } \\
\end{array}$} & \multirow[b]{2}{*}{$\begin{array}{l}\text { Speed } \\
\text { (kts) }\end{array}$} & \multirow[b]{2}{*}{ Direction } \\
\hline & & & & Heating & Cooling & & & & \\
\hline 1 & 79 & 52 & 66 & $\ldots$ & 1 & $T$ & 0 & 13 & 36 \\
\hline 2 & 82 & 59 & 71 & - & 6 & 0.00 & 0 & 21 & 02 \\
\hline 3 & 88 & 54 & 71 & $\ldots$ & 6 & 0.00 & 0 & 14 & $35,36,01$ \\
\hline 4 & 82 & 57 & 70 & $\ldots$ & 5 & 0.00 & 0 & 13 & 21 \\
\hline 5 & 84 & 66 & 75 & $\cdots$ & 10 & 0.09 & 0 & 15 & 31 \\
\hline 6 & 84 & 63 & 74 & $\ldots$ & 9 & 0.00 & 0 & 10 & 08 \\
\hline 7 & 68 & 61 & 65 & $\ldots$ & $\ldots$ & 0.39 & 0 & 14 & 05 \\
\hline 8 & 65 & 60 & 63 & 2 & - & 0.16 & 0 & 12 & 04,05 \\
\hline 9 & 79 & 61 & 70 & $\ldots$ & 5 & $T$ & 0 & 14 & 33 \\
\hline 10 & 81 & 59 & 70 & $\ldots$ & 5 & 0.29 & 0 & 17 & 30 \\
\hline 11 & 73 & 55 & 64 & 1 & $\ldots$ & 0.00 & 0 & 11 & 28 \\
\hline 12 & 72 & 55 & 64 & 1 & $\ldots$ & 0.00 & 0 & 13 & 10,06 \\
\hline 13 & 61 & 57 & 59 & 6 & $\ldots$ & 0.24 & 0 & 16 & 05 \\
\hline 14 & 72 & 55 & 64 & 1 & $\ldots$ & 0.14 & 0 & 17 & 06 \\
\hline 15 & 74 & 50 & 62 & 3 & - & 0.00 & 0 & 18 & 22,23 \\
\hline 16 & 70 & 54 & 62 & 3 & $\ldots$ & $T$ & 0 & 13 & 23 \\
\hline 17 & 59 & 52 & 56 & 9 & $\ldots$ & 0.71 & 0 & 21 & 02,01 \\
\hline 18 & 61 & 52 & 57 & 8 & - & $2.26^{*}$ & 0 & 28 & 36 \\
\hline 19 & 72 & 45 & 59 & 6 & $\ldots$ & 0.00 & 0 & 24 & 35 \\
\hline 20 & 75 & 39 & 57 & 8 & -- & 0.00 & 0 & 16 & 20 \\
\hline 21 & 77 & 41 & 59 & 6 & -- & 0.00 & 0 & 18 & $28,24,26$ \\
\hline 22 & 63 & 46 & 52 & 13 & $\ldots$ & $1.42^{*}$ & 0 & 11 & 16,32 \\
\hline 23 & 61 & 50 & 56 & 9 & $\ldots$ & 0.57 & 0 & 17 & $01,33,36$ \\
\hline 24 & 59 & 36 & 48 & 17 & $\ldots$ & 0.14 & 0 & 11 & 14 \\
\hline 25 & 64 & 43 & 54 & 11 & $\ldots$ & 0.04 & 0 & 20 & 31 \\
\hline 26 & 63 & 37 & 50 & 15 & $\ldots$ & 0.00 & 0 & 15 & 36 \\
\hline 27 & 63 & 41 & 52 & 13 & $\ldots$ & $T$ & 0 & 14 & $16,17,19,20$ \\
\hline 28 & 72 & 57 & 65 & -- & -- & 0.67 & 0 & 25 & 21 \\
\hline 29 & 66 & 46 & 56 & 9 & $\ldots$ & 0.53 & 0 & 9 & 19,24 \\
\hline 30 & 66 & 43 & 55 & 10 & $\ldots$ & 0.00 & 0 & 18 & 30 \\
\hline 31 & $\ldots$ & $\ldots$ & $\ldots$ & - & $\ldots$ & - & $\ldots$ & $\ldots$ & $\ldots$ \\
\hline Total & $\ldots$ & $\ldots$ & $\ldots$ & 127 & 47 & 7.69 & 0 & -- & $\ldots$ \\
\hline Mean & 71.2 & 51.5 & 61.5 & $\ldots$ & $\ldots$ & $\ldots$ & $\ldots$ & $\ldots$ & $\ldots$ \\
\hline Extreme & 88 & 36 & $\ldots$ & -- & -- & 2.26 & 0 & 28 & 36 \\
\hline
\end{tabular}




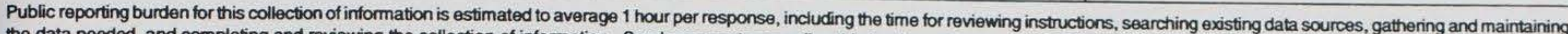

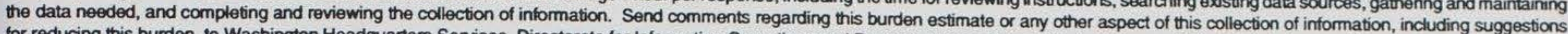

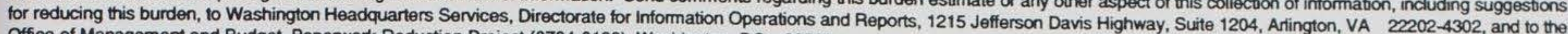
Office of Management and Budget, Paperwork Reduction Project (0704-0188), Washington, DC 20503.
1. AGENCY USE ONLY (Leave blank)
2. REPORT DATE July 1997

\section{REPORT TYPE AND DATES COVERED} Final report

4. TITLE AND SUBTITLE

Leaky Coaxial Cable Sensor Slot/Sealant Configuration Performance Monitoring

\section{FUNDING NUMBERS}

6. AUTHOR(S)

Larry N. Lynch

7. PERFORMING ORGANIZATION NAME(S) AND ADDRESS(ES)

U.S. Army Engineer Waterways Experiment Station

3909 Halls Ferry Road, Vicksburg, MS 39180-6199

8. PERFORMING ORGANIZATION REPORT NUMBER

Miscellaneous Paper GL-97-11

9. SPONSORING/MONITORING AGENCY NAME(S) AND ADDRESS(ES)

Department of the Air Force

Electronic Security and Communications Center of Excellence

Hanscom Air Force Base, Massachusetts 01731

11. SUPPLEMENTARY NOTES

Available from National Technical Information Service, 5285 Port Royal Road, Springfield, VA 22161.

12a. DISTRIBUTIONAVAILABILITY STATEMENT

Approved for public release; distribution is unlimited.

12b. DISTRIBUTION CODE

13. ABSTRACT (Maximum 200 words)

A new slot/sealant configuration has been recommended for the installation of leaky coaxial cable sensor systems. This report documents the field performance of a trial installation at Westover Air Force Base, Massachusetts. Visual observations after 2 years of service indicate that the new slot/sealant configuration is performing satisfactorily.

14. SUBJECT TERMS

Field performance

Leaky coaxial cable sensors

15. NUMBER OF PAGES

Silicone sealants

46

16. PRICE CODE
1 OF REPORT

UNCLASSIFIED
18. SECURITY CLASSIFICATION OF THIS PAGE UNCLASSIFIED
19. SECURITY CLASSIFICATION OF ABSTRACT 